\title{
Connecting orbits and invariant manifolds in the spatial restricted three-body problem
}

\author{
G Gómez ${ }^{1}$, W S Koon ${ }^{2,5}$, M W Lo ${ }^{3}$, J E Marsden ${ }^{2}$, J Masdemont ${ }^{4}$ and \\ S D Ross ${ }^{2}$ \\ ${ }^{1}$ Departament de Matemàtica Aplicada i Anàlisi, Universitat de Barcelona, Barcelona, Spain \\ 2 Control and Dynamical Systems, California Institute of Technology, MC 107-81, Pasadena, \\ CA 91125, USA \\ ${ }^{3}$ Navigation and Mission Design, Jet Propulsion Laboratory, California Institute of Technology, \\ M/S 301-140L, Pasadena, CA 91109, USA \\ ${ }^{4}$ Departament de Matemàtica Aplicada I, Universitat Politècnica de Catalunya, Barcelona, Spain \\ E-mail: gerard@maia.ub.es, koon@cds.caltech.edu, martin.lo@jpl.nasa.gov, \\ marsden@cds.caltech.edu, josep@barquins.upc.es and shane@cds.caltech.edu
}

Received 21 August 2003, in final form 24 March 2004

Published 27 May 2004

Online at stacks.iop.org/Non/17/1571

DOI: 10.1088/0951-7715/17/5/002

Recommended by A Chenciner

\begin{abstract}
The invariant manifold structures of the collinear libration points for the restricted three-body problem provide the framework for understanding transport phenomena from a geometrical point of view. In particular, the stable and unstable invariant manifold tubes associated with libration point orbits are the phase space conduits transporting material between primary bodies for separate three-body systems. These tubes can be used to construct new spacecraft trajectories, such as a 'Petit Grand Tour' of the moons of Jupiter. Previous work focused on the planar circular restricted three-body problem. This work extends the results to the three-dimensional case.

Besides providing a full description of different kinds of libration motions in a large vicinity of these points, this paper numerically demonstrates the existence of heteroclinic connections between pairs of libration orbits, one around the libration point $L_{1}$ and the other around $L_{2}$. Since these connections are asymptotic orbits, no manoeuvre is needed to perform the transfer from one libration point orbit to the other. A knowledge of these orbits can be very useful in the design of missions such as the Genesis Discovery Mission, and may provide the backbone for other interesting orbits in the future.
\end{abstract}

PACS numbers: 45.10.-b, 45.20.Jj, 45.50.Jf, 45.50.Pk, 95.10.Ce, 95.10.Fh

(Some figures in this article are in colour only in the electronic version)

5 Author to whom any correspondence should be addressed. 


\section{Introduction}

New space missions are increasingly more complex, requiring new and unusual kinds of orbits to meet their scientific goals, orbits which are not easily found by the traditional conic approach. The delicate heteroclinic dynamics employed by the Genesis Discovery Mission dramatically illustrates the need for a new paradigm: study of the three-body problem using dynamical systems theory as laid out by Poincaré $[16,20,26]$.

The dynamical structures of the three-body problem (such as stable and unstable manifolds, and bounding surfaces), reveal much about the morphology and transport of particles within the solar system, be they asteroids, dust grains, or spacecraft. The cross-fertilization between the study of the natural dynamics in the solar system and engineering applications has produced a number of new techniques for constructing spacecraft trajectories with desired behaviour, such as rapid transition between the interior and exterior Hill's regions, resonance hopping, and temporary capture [27].

The invariant manifold structures associated with the collinear libration points for the restricted three-body problem, which exist for a range of energies, provide a framework for understanding the aforementioned dynamical phenomena from a geometrical point of view. In particular, the stable and unstable invariant manifold tubes associated with bounded orbits around the libration points $L_{1}$ and $L_{2}$ are phase space structures that conduct particles to and from the smaller primary body (e.g. Jupiter in the Sun-Jupiter-comet three-body system), and between primary bodies for separate three-body systems [28], e.g. Saturn and Jupiter in the Sun-Saturn-comet and the Sun-Jupiter-comet three-body systems.

Petit Grand Tour of Jovian moons. The invariant manifold tubes can be used to produce new techniques for constructing spacecraft trajectories with interesting characteristics. These may include mission concepts such as a low energy transfer from the Earth to the Moon [29] and a 'Petit Grand Tour' of the moons of Jupiter, described below and in [30].

Using the phase space tubes in each three-body system, we are able to construct a transfer trajectory from the Earth which executes an unpropelled (i.e. ballistic) capture at the Moon. An Earth-to-Moon trajectory of this type, which utilizes the perturbation by the Sun, requires less fuel than a transfer trajectory using segments of Keplerian motion, i.e. the 'patched-conics' approach [2].

Similarly, by approximating a spacecraft's motion in the $(n+1)$-body gravitational field of Jupiter and $n$ of its planet-sized moons into several segments of purely three-body motion-involving Jupiter, the $i$ th moon, and the spacecraft—we can design a trajectory for the spacecraft which follows a prescribed itinerary in visiting the $n$ moons. In an earlier study of a transfer from Ganymede to Europa [30], we found our fuel consumption for impulsive burns, as measured by the total norm of velocity displacements, $\Delta V$, to be less than half the Hohmann transfer value. We found this to be the case for the following example tour: starting beyond Ganymede's orbit, the spacecraft is ballistically captured by Ganymede, orbits it once, escapes in the direction of Europa, and ends in a ballistic capture at Europa.

One advantage of this Petit Grand Tour as compared with the Voyager-type flybys is the 'leap-frogging' strategy. In this new approach to mission design, the spacecraft can orbit a moon in a loose temporary capture orbit for a desired number of circuits, escape the moon, and then perform a transfer $\Delta V$ to become ballistically captured by a nearby moon for some number of orbits about that moon, etc. Instead of brief flybys lasting only seconds, a scientific spacecraft can orbit several different moons for any desired duration. Furthermore, the total $\Delta V$ necessary is much less than that necessary using purely two-body motion segments. 

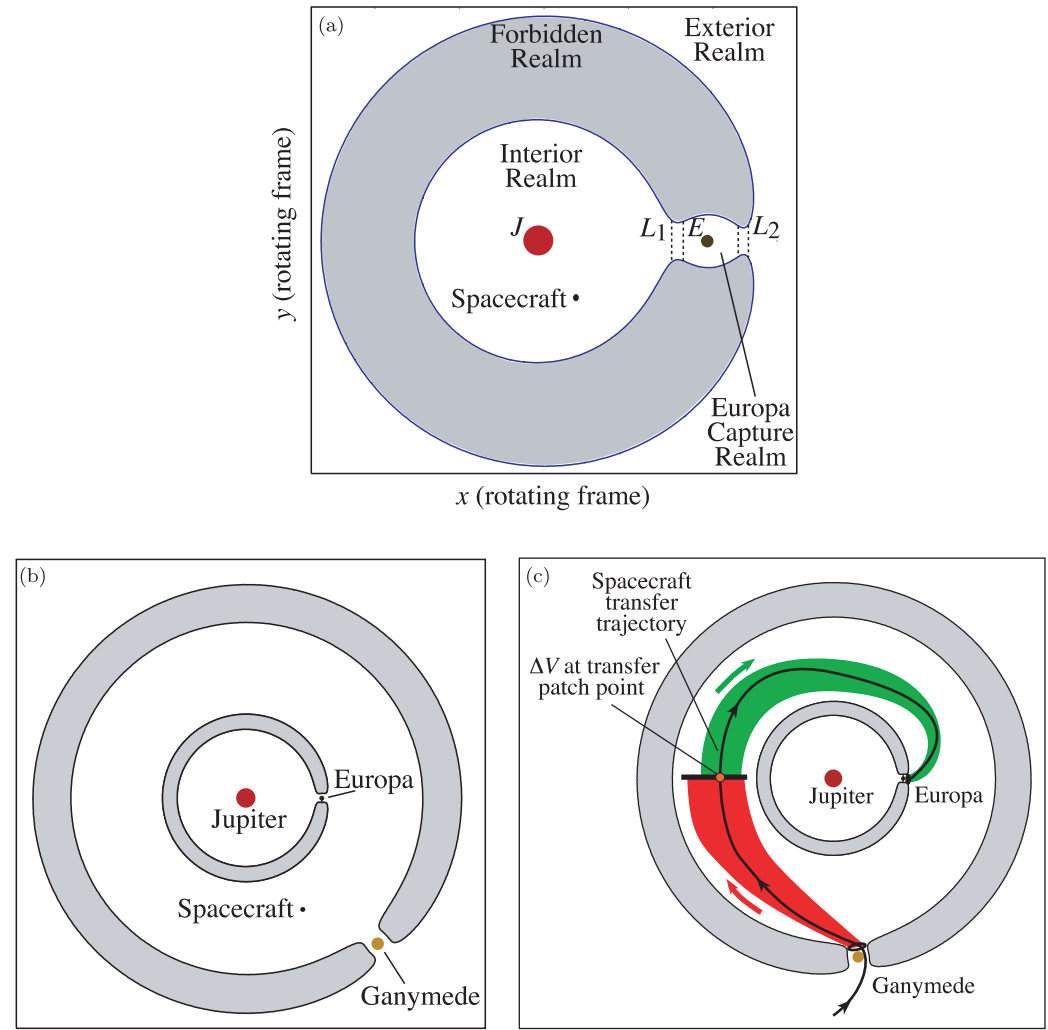

Figure 1. The patched three-body model. (a) The co-orbiting frame with Europa is shown, otherwise known as the rotating frame. The spacecraft's motion in each Jupiter-Moon-spacecraft rotating frame is limited to the region in white due to constant energy in that system (constant Jacobi integral). We work with three-body energy regimes where the region surrounding the moon's orbit (shaded) is energetically forbidden to spacecraft motion. Note the small opening near the moon, permitting capture and escape. $(b)$ The four-body system approximated as two nested three-body systems: this picture is only a schematic, as the spacecraft's motion conserves the Jacobi integral in only one system at a time. (c) We seek an intersection between the dynamical channel enclosed by Ganymede's $L_{1}$ periodic orbit unstable manifold and the dynamical channel enclosed by Europa's $L_{2}$ periodic orbit stable manifold (shown in schematic). Integrate forward and backward from the patch point (with $\Delta V$ to take into account velocity discontinuity) to generate the desired transfer trajectory between the moons.

The design of the Petit Grand Tour in the planar case is guided by two main ideas (see [30]).

(i) The motion of the spacecraft in the gravitational field of the three bodies Jupiter, Ganymede and Europa is approximated by two segments of purely three-body motion in the planar, circular, restricted three-body model. The trajectory segment in the first three-body system, Jupiter-Ganymede-spacecraft, is appropriately patched to the segment in the Jupiter-Europa-spacecraft three-body system.

(ii) For each segment of purely three-body motion, the invariant manifold tubes of $L_{1}$ and $L_{2}$ periodic orbits (p.o.) leading towards or away from temporary capture around a moon, as in figure 1 , are used to construct an orbit with the desired behaviour. This initial solution is then refined to obtain a trajectory in a more accurate four-body model.

The patched three-body model considers the motion of a particle (or spacecraft) in the field of $n$ bodies, considered two at a time, e.g. Jupiter and its $i$ th moon, $M_{i}$. When the trajectory of 
a spacecraft comes close to the orbit of $M_{i}$, the perturbation of the spacecraft's motion away from purely Keplerian motion about Jupiter is dominated by $M_{i}$. In this situation, we say that the spacecraft's motion is well modelled by the Jupiter- $M_{i}-$ spacecraft restricted three-body problem.

Switching orbit. There comes a point along the spacecraft's trajectory at which a rocket burn manoeuvre-effecting a change in velocity of magnitude $\Delta V$-will make the spacecraft's perturbation switch from being dominated by $M_{i}$ to being dominated by another moon, $M_{k}$. The set of possible 'switching orbits', which we will refer to as the switching region, is the analogue to the 'sphere of influence' concept used in the patched-conic approach to trajectory design, which guides a mission designer in determining when to switch the central body for the model of the spacecraft's Keplerian motion. In this paper, Jupiter is always considered, but one switches the third body in the three-body model from $M_{i}$ to $M_{k}$.

Our goal is to find piecewise continuous trajectories in the phase space which lead a spacecraft from a loose orbit about $M_{i}$ to a loose orbit about $M_{k}$, trajectories continuous in position, but allowing for discontinuities in the velocity, for which impulsive rocket burns will be necessary. We refer to the phase space of these loose orbits as the 'capture realm'. In the procedure we outline, we seek intersections between invariant manifold 'tubes' which connect the capture realm of one moon with that of another moon. In the planar case, these solid tubes are bounded by stable and unstable invariant manifold tubes of $L_{1}$ and $L_{2}$ p.o.'s, which act as separatrices separating transit orbits from non-transit orbits. Transit orbits lead towards or away from a capture realm, whereas non-transit orbits do not. The stable and unstable manifolds of $L_{1}$ and $L_{2}$ p.o.'s are the phase space structures that provide a conduit for orbits between realms within each three-body system as well as between capture realms surrounding primary bodies for separate three-body systems [27].

Extending results from planar model to spatial model. Previous work based on the planar circular restricted three-body problem (PCR3BP) revealed the basic structures controlling the dynamics [27-30]. But current missions such as the Genesis Discovery Mission [20,21], and future missions will require three-dimensional capabilities, such as control of the latitude and longitude of a spacecraft's escape from and entry into a planetary or moon orbit. For example, a future mission to send a probe to orbit Europa may desire a capture into a high inclination polar orbit around Europa [38,31,37,41]. Three-dimensional capability is also required when decomposing a multibody system into three-body subsystems which are not co-planar, such as the Earth-Sun-spacecraft and Earth-Moon-spacecraft systems. (The tilt in the orbital planes of the Earth around the sun and the moon around the Earth is about $5^{\circ}$.) These demands necessitate dropping the restriction to planar motion, and extension of earlier results to the spatial model (CR3BP).

In our current work on the spatial three-body problem (also see [13]), we show that the invariant manifold structures of the collinear libration points still act as the separatrices between two types of motion: (i) inside the invariant manifold tubes, the motion consists of a transit through a neck, a set of paths called transit orbits; (ii) outside the tubes, no such transit motion is possible. We design an algorithm for constructing orbits with any prescribed itinerary and obtain some initial results for a basic itinerary. Furthermore, we apply these new techniques to the construction of a three-dimensional Petit Grand Tour of the Jovian moon system. By approximating the dynamics of the Jupiter-Europa-Ganymede-spacecraft fourbody problem as two three-body subproblems, we seek intersections (in position space only) between the tubes of transit orbits enclosed by the stable and unstable manifold tubes. As shown 
(a)

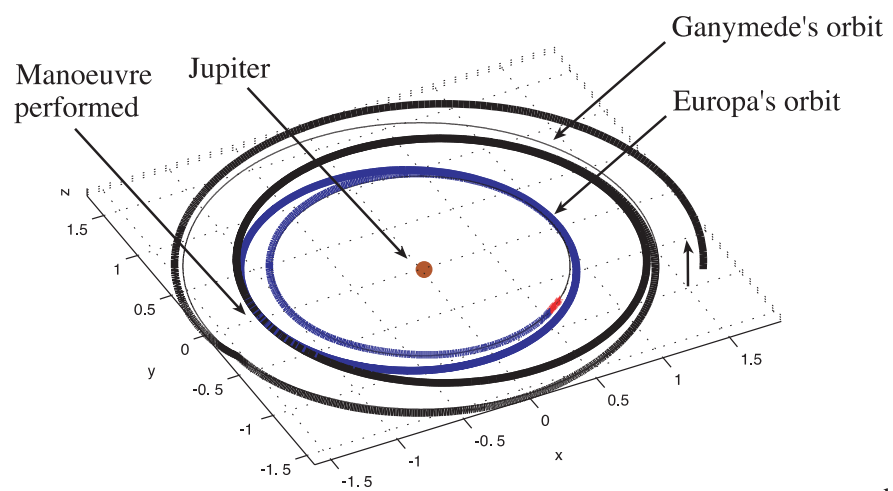

Injection into high inclination

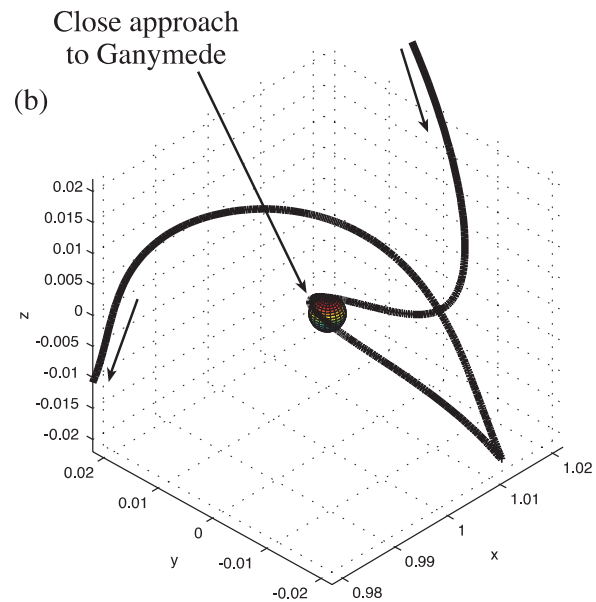

(c)

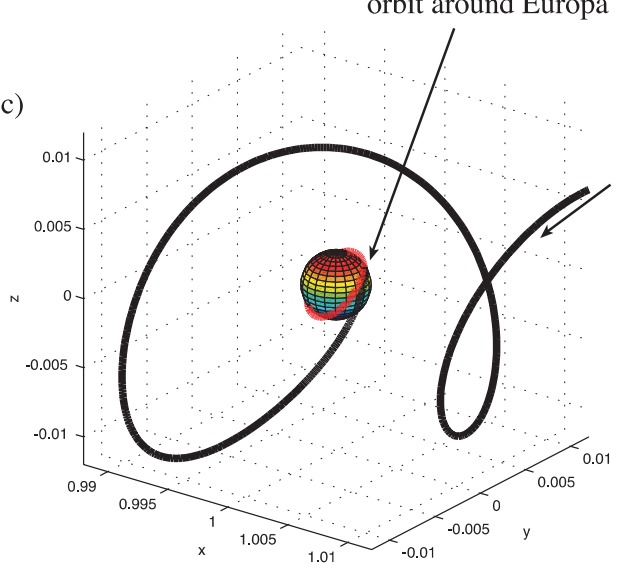

Figure 2. The three-dimensional Petit Grand Tour space mission concept for the Jovian moons. (a) We show a spacecraft trajectory coming into the Jupiter system and transferring from Ganymede to Europa using a single impulsive manoeuvre, shown in a Jupiter-centred inertial frame. (b) The spacecraft performs one loop around Ganymede, using no propulsion at all, as shown here in the Jupiter-Ganymede rotating frame. (c) The spacecraft arrives in Europa's vicinity at the end of its journey and performs a final propulsion manoeuvre to get into a high inclination circular orbit around Europa, as shown here in the Jupiter-Europa rotating frame.

in figure 2, we design a sample low energy transfer trajectory from an initial Jovian insertion trajectory, leading to Ganymede and finally to Europa, ending in a high inclination orbit around Europa.

Heteroclinic connections between libration orbits. Besides stable and unstable manifold tubes, centre manifolds of the collinear libration points have played a very important role in space mission design for a long time. Since 1978, when NASA launched the ISEE-3 spacecraft $[7,36]$, Lissajous and halo type trajectories around the collinear libration points have been considered in the trajectory design of many space missions. The SOHO spacecraft has used a halo orbit around $L_{1}$ in the Earth-Sun system as a nominal station orbit since 1996. In the near future, the European Space Agency is considering two missions to $L_{2}$ in the Sun-Earth system, FIRST and PLANK. Furthermore, for NASA's Next Generation Space Telescope, the follow-on to the Hubble Telescope, an $L_{2}$ orbit is also being considered. All this interest in libration point orbits justifies the study of the dynamics around an extended neighbourhood of these points in order that more complex missions can be envisaged. 
In this paper, besides providing a full description of different kinds of libration motions in a large vicinity of these points, we show the existence of heteroclinic connections between pairs of libration orbits, one around the libration point $L_{1}$ and the other around $L_{2}$. Since these connections are asymptotic orbits, no manoeuvre is needed to perform the transfer from one libration orbit to the other. Knowledge of these orbits could be very useful in the design of missions such as Genesis [21], and may inspire the use of similar orbits in the future.

Computation of the centre manifold and its stable and unstable manifolds. It is well known that the linear behaviour of collinear libration points is of the type saddle $\times$ centre $\times$ centre. This behaviour is inherited by the libration orbits, all of which are highly unstable $[10,11,39]$. Hence, numerical exploration in the neighbourhood of the libration points is not straightforward for two reasons. The first one is the high dimensionality of the problem (six phase space dimensions), which makes the exploration time consuming, even using simple models like the CR3BP. The second reason is the highly unstable character of the solutions near the libration points. Due to this instability, errors in the initial conditions multiply by a factor of the order of 1500 every half revolution of the secondary around the primary, making it difficult to get an idea of the flow and the orbits in the vicinity of these points.

However, the instability can be handled and the dimensionality reduced by a procedure called reduction to the centre manifold, to be introduced shortly (see also $[10,23]$ ). The fundamental idea is based on canonical transformations of the Hamiltonian equations by the Lie series method, implemented in a different way from the 'standard' procedure introduced in [6]. The change of variables allows us to have a two degree of freedom Hamiltonian containing only the orbits in the centre manifold. Roughly speaking, this means we remove the main instability. For each level of energy, the orbits are in a three-dimensional manifold that can be represented and viewed globally in a two-dimensional Poincaré surface of section.

The procedure gives a complete description of the libration orbits around an extended neighbourhood of the collinear libration points. The main objects found are: planar and vertical families of Lyapunov p.o.; Lissajous orbits; periodic halo orbits; and quasi-halo orbits. An approach that is not entirely numerical in nature has been used to compute these orbits in the past; starting from the equations of motion of the CR3BP and implementing semi-analytic procedures based on asymptotic series of the Lindsted-Poincaré type [10, 17, 18,23].

In this paper, we implement a slightly different procedure for the computation of the reduction to the centre manifold, in order to have all the possible initial conditions in the centre manifold of a selected level of energy (i.e. the Jacobi constant of the CR3BP). The connections between the orbits of $L_{1}$ and $L_{2}$ are constructed looking for the intersections of the unstable manifold of a libration orbit around $L_{i}$ with the stable one of a libration one around $L_{3-i}$ for $i=1,2$. The orbits are asymptotic to both libration orbits in the same level of energy and thus, in the ideal situation of the CR3BP, no $\Delta V$ is required to perform the transfer from one orbit to the other.

This efficient way of computing stable and unstable manifolds for the centre manifold of a selected level of energy allows us to construct the Petit Grand Tour of Jovian moons.

Similarity between celestial mechanics and chemical dynamics. We note that there is some overlap between the mathematical problem considered here and the chemical dynamics problem considered by Uzer et al in [40]. This is due to the considerable mathematical similarities between some problems in celestial mechanics and problems in chemical dynamics, which has been noted previously in a successful application of ideas from chemistry to the problem of asteroid escape rates [22]. However, due to the different physical focus of these 
two communities, the specific questions that are posed and the results that are presented can be quite different. A key difference between this paper and [40] is that we globalize the stable and unstable manifold tubes far from the vicinity of the equilibrium points, with the goal of constructing individual trajectories that traverse more than one 'potential barrier', to use chemical dynamics terminology. In [40], the main concern is with the computation of chemical reaction rates.

Structure of the paper. In sections 2 through 4, we review some known mathematical and numerical results which guide the numerical explorations of sections 5 through 8 . In the appendix, we give a description of the software used for the numerical explorations.

\section{Circular restricted three-body problem}

The orbital planes of Ganymede and Europa are within $0.3^{\circ}$ of each other, and their orbital eccentricities are 0.0006 and 0.0101 , respectively. Furthermore, since the masses of both moons are small, and they are on rather distant orbits, the patched spatial CR3BP is an excellent starting model for illuminating the transfer dynamics between these moons. We assume the orbits of Ganymede and Europa are co-planar, but the spacecraft is not restricted to their common orbital plane.

The spatial circular restricted three-body problem. We begin by recalling the equations for the CR3BP. The two main bodies, which we call generically Jupiter (denoted $J$ ) and the moon (denoted $M$ ), have a total mass that is normalized to one. Their masses are denoted by $m_{J}=1-\mu$ and $m_{M}=\mu$, respectively (see figure $3(a)$ ). These bodies rotate in the plane counterclockwise about their common centre of mass and with the angular velocity normalized to one. The third body, which we call the spacecraft, is free to move in threedimensional space and its motion is assumed not to affect the primaries. Note that the mass parameters for the Jupiter-Ganymede and Jupiter-Europa systems are $\mu_{G}=7.802 \times 10^{-5}$ and $\mu_{E}=2.523 \times 10^{-5}$, respectively.

Choose a rotating coordinate system so that the origin is at the centre of mass and Jupiter and the moon are fixed on the $x$-axis at $(-\mu, 0,0)$ and $(1-\mu, 0,0)$, respectively (see figure $3(a))$. Let $(x, y, z)$ be the position of the spacecraft in the rotating frame.

Equations of motion. There are several ways to derive the equations of motion for this system. An efficient technique is to use the covariance of the Lagrangian formulation and use the Lagrangian directly in a moving frame (see [32]). This method gives the equations in Lagrangian form. Then, the equations of motion of the spacecraft can be written in second-order form as

$$
\ddot{x}-2 \dot{y}=\Omega_{x}, \quad \ddot{y}+2 \dot{x}=\Omega_{y}, \quad \ddot{z}=\Omega_{z},
$$

where

$$
\Omega(x, y, z)=\frac{x^{2}+y^{2}}{2}+\frac{1-\mu}{r_{1}}+\frac{\mu}{r_{2}}+\frac{\mu(1-\mu)}{2}
$$

and $\Omega_{x}, \Omega_{y}$, and $\Omega_{z}$ are the partial derivatives of $\Omega$ with respect to the variables $x, y$ and $z$. Also, $r_{1}=\sqrt{(x+\mu)^{2}+y^{2}+z^{2}}, r_{2}=\sqrt{(x-1+\mu)^{2}+y^{2}+z^{2}}$. This form of the equations of motion has been studied in detail (see [39]) and they are called the equations of the CR3BP. 

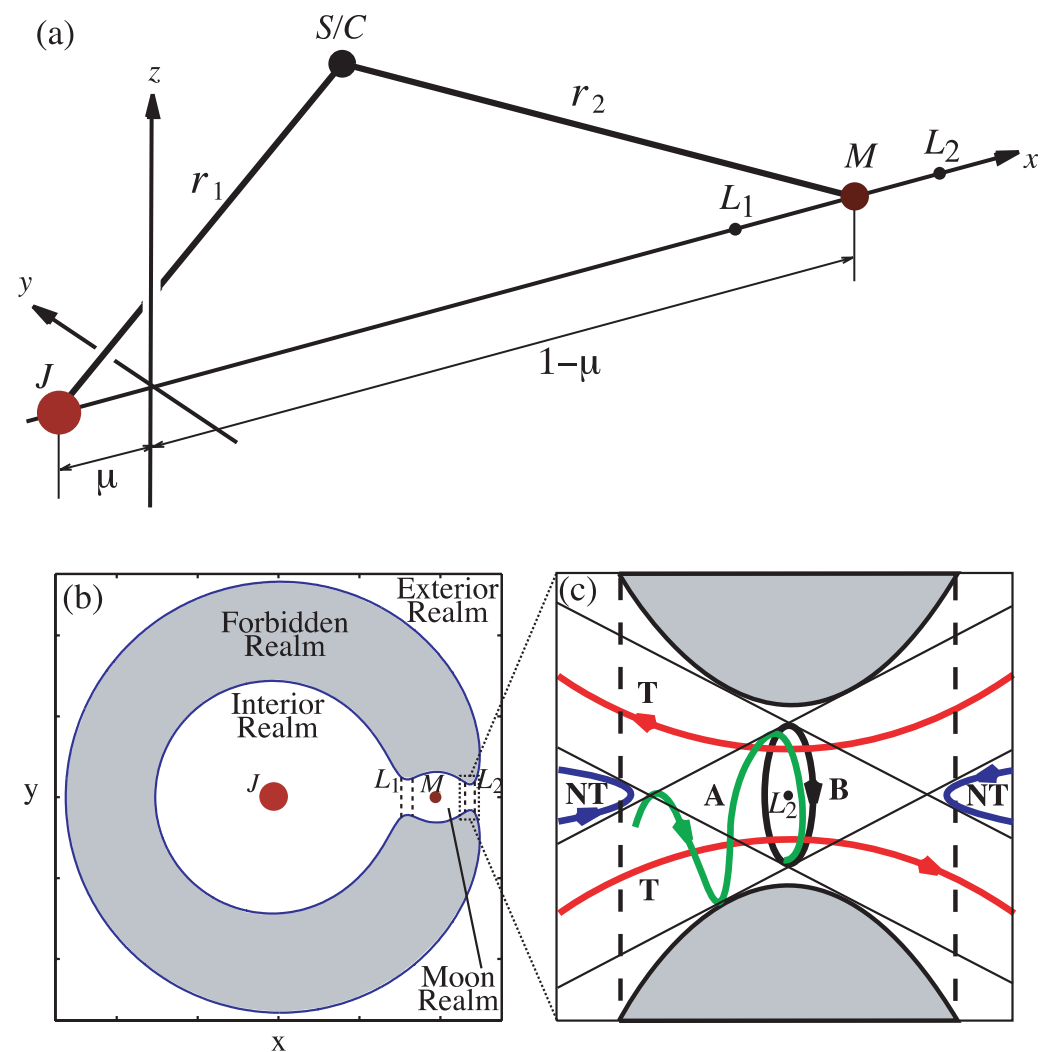

Figure 3. (a) Equilibrium points of the CR3BP as viewed, not in any inertial frame, but in the rotating frame, where Jupiter and a Jovian moon are at fixed positions along the $x$-axis. (b) Projection of the three-dimensional Hill's region on the $(x, y)$-plane (schematic, the region in white), which contains three large realms connected by neck regions about $L_{1}$ and $L_{2}$. (c) The flow in the $L_{2}$ neck region is shown projected on the $(x, y)$-plane, showing a bounded orbit around $L_{2}$ (labelled B), an asymptotic orbit winding onto the bounded orbit (A), two transit orbits (T) and two non-transit orbits (NT), shown schematically. A similar figure holds for the region around $L_{1}$.

After applying the Legendre transformation to the Lagrangian formulation, one finds that the Hamiltonian function is given by

$$
H=\frac{1}{2}\left\{\left(p_{x}+y\right)^{2}+\left(p_{y}-x\right)^{2}+p_{z}^{2}\right\}-\Omega(x, y, z) .
$$

Therefore, Hamilton's equations are given by

$$
\begin{aligned}
& \dot{x}=\frac{\partial H}{\partial p_{x}}=p_{x}+y, \quad \dot{p}_{x}=-\frac{\partial H}{\partial x}=p_{y}-x+\Omega_{x}, \\
& \dot{y}=\frac{\partial H}{\partial p_{y}}=p_{y}-x, \quad \dot{p}_{y}=-\frac{\partial H}{\partial y}=-p_{x}-y+\Omega_{y}, \\
& \dot{z}=\frac{\partial H}{\partial p_{z}}=p_{z}, \quad \dot{p}_{z}=-\frac{\partial H}{\partial z}=\Omega_{z} .
\end{aligned}
$$

Jacobi integral. The system (1) has a first integral called the Jacobi integral, which is given by

$$
C(x, y, z, \dot{x}, \dot{y}, \dot{z})=-\left(\dot{x}^{2}+\dot{y}^{2}+\dot{z}^{2}\right)+2 \Omega(x, y, z)=-2 E(x, y, z, \dot{x}, \dot{y}, \dot{z}) .
$$

We shall use $E$ when we regard the Hamiltonian as a function of the positions and velocities and $H$ when we regard it as a function of the positions and momenta. 
Equilibrium points and Hill's regions. The system (1) has five equilibrium points, all of which are in the $(x, y)$ plane: three collinear points on the $x$-axis, called $L_{1}, L_{2}, L_{3}$ (see figure $3(a)$ ) and two equilateral points called $L_{4}$ and $L_{5}$. These equilibrium points are critical points of the (effective potential) function $\Omega$. The value of the Jacobi integral at the point $L_{i}$ will be denoted by $C_{i}$.

The level surfaces of the Jacobi constant, which are also energy surfaces, are invariant five-dimensional manifolds. Let $\mathcal{H}$ be that energy surface, i.e.

$$
\mathcal{H}(\mu, C)=\{(x, y, z, \dot{x}, \dot{y}, \dot{z}) \mid C(x, y, z, \dot{x}, \dot{y}, \dot{z})=\text { constant }\}
$$

The projection of this surface onto position space is called a Hill's region

$$
H(\mu, C)=\left\{(x, y, z) \mid \Omega(x, y, z) \geqslant \frac{C}{2}\right\} .
$$

The boundary of $H(\mu, C)$ is the zero velocity surface. The intersection of this surface with the $(x, y)$-plane is the zero velocity curve. The spacecraft can move only within this region. Our main concern here is the behaviour of the orbits of equations (1) whose Jacobi constant is just below that of $L_{2}$; that is, $C<C_{2}$. For this case, the three-dimensional Hill's region is partitioned into three large realms connected by neck regions about $L_{1}$ and $L_{2}$, as shown in figure 3(b). Thus, orbits with a Jacobi constant just below that of $L_{2}$ are energetically permitted to make a transit through the two neck regions from the interior realm (inside the moon's orbit) to the exterior realm (outside the moon's orbit) passing through the moon (capture) realm.

\section{Invariant manifold as separatrix}

Studying the linearization of the dynamics near the equilibria is of course an essential ingredient for understanding the more complete nonlinear dynamics [1,3, 27, 33]. We refer to the neighbourhood of the equilibrium point $L_{i}$ restricted to an energy surface as the equilibrium region, $\mathcal{R}_{i}$. It can be shown that for a value of the Jacobi constant just below that of $L_{1}$ (respectively $L_{2}$ ), the nonlinear dynamics in the equilibrium region $\mathcal{R}_{1}$ (respectively $\mathcal{R}_{2}$ ) is qualitatively the same as the linearized picture that we will describe later. For details, see the appendix at the end of this paper as well as other references [15,23,19,43]. This geometric insight will be used later to guide our numerical explorations in constructing orbits with prescribed itineraries.

Linearization near the collinear equilibria. We will denote by $(k, 0,0,0, k, 0)$ the positions and momenta of any of the collinear libration points. To find the linearized equations, we need the quadratic terms of the Hamiltonian $H$ in equation (2) as expanded about $(k, 0,0,0, k, 0)$. After making a coordinate change with $(k, 0,0,0, k, 0)$ as the origin, these quadratic terms form the Hamiltonian function for the linearized equations, which we shall call $\mathrm{H}_{2}$

$$
H_{2}=\frac{1}{2}\left\{\left(p_{x}+y\right)^{2}+\left(p_{y}-x\right)^{2}+p_{z}^{2}-a x^{2}+b y^{2}+c z^{2}\right\}
$$

where, $a, b$ and $c$ are defined by $a=2 c+1, b=c-1$, and where

$$
c=\mu|k-1+\mu|^{-3}+(1-\mu)|k+\mu|^{-3} \text {. }
$$

A short computation gives the linearized equations in the form

$$
\begin{array}{rlrl}
\dot{x} & =\frac{\partial H_{2}}{\partial p_{x}}=p_{x}+y, & \dot{p}_{x}=-\frac{\partial H_{2}}{\partial x}=p_{y}-x+a x, \\
\dot{y}=\frac{\partial H_{2}}{\partial p_{y}}=p_{y}-x, & \dot{p}_{y}=-\frac{\partial H_{2}}{\partial y}=-p_{x}-y-b y, \\
\dot{z}=\frac{\partial H_{2}}{\partial p_{z}}=p_{z}, & \dot{p}_{z}=-\frac{\partial H_{2}}{\partial z}=-c z .
\end{array}
$$


It is straightforward to show that the eigenvalues of this linear system have the form $\pm \lambda, \pm \mathrm{i} v$ and $\pm \mathrm{i} \omega$, where $\lambda, v$ and $\omega$ are positive constants and $\nu \neq \omega$.

To better understand the orbit structure on the phase space, we make a linear change of coordinates with the eigenvectors as the axes of the new system. Using the corresponding new coordinates $q_{1}, p_{1}, q_{2}, p_{2}, q_{3}, p_{3}$, the differential equations assume the simple form

$$
\begin{array}{ll}
\dot{q}_{1}=\lambda q_{1}, & \dot{p}_{1}=-\lambda p_{1}, \\
\dot{q}_{2}=v p_{2}, & \dot{p}_{2}=-v q_{2}, \\
\dot{q}_{3}=\omega p_{3}, & \dot{p}_{3}=-\omega q_{3}
\end{array}
$$

and the Hamiltonian function becomes

$$
H_{2}=\lambda q_{1} p_{1}+\frac{v}{2}\left(q_{2}^{2}+p_{2}^{2}\right)+\frac{\omega}{2}\left(q_{3}^{2}+p_{3}^{2}\right)
$$

The solutions of equations (3) can be conveniently written as

$$
\begin{aligned}
& q_{1}(t)=q_{1}^{0} \mathrm{e}^{\lambda t}, \quad p_{1}(t)=p_{1}^{0} \mathrm{e}^{-\lambda t}, \\
& q_{2}(t)+\mathrm{i} p_{2}(t)=\left(q_{2}^{0}+\mathrm{i} p_{2}^{0}\right) \mathrm{e}^{-\mathrm{i} v t}, \\
& q_{3}(t)+\mathrm{i} p_{2}(t)=\left(q_{3}^{0}+\mathrm{i} p_{3}^{0}\right) \mathrm{e}^{-\mathrm{i} \omega t},
\end{aligned}
$$

where the constants $q_{1}^{0}, p_{1}^{0}, q_{2}^{0}+\mathrm{i} p_{2}^{0}$ and $q_{3}^{0}+\mathrm{i} p_{3}^{0}$ are the initial conditions. These linearized equations admit integrals in addition to the Hamiltonian function; namely, the functions $q_{1} p_{1}$, $q_{2}^{2}+p_{2}^{2}$ and $q_{3}^{2}+p_{3}^{2}$ are constant along solutions.

The linearized phase space. For positive $h$ and $c$, the region $\mathcal{R}$ (referring to either $\mathcal{R}_{1}$ or $\mathcal{R}_{2}$ ), is determined by

$$
H_{2}=h \quad \text { and } \quad\left|p_{1}-q_{1}\right| \leqslant c .
$$

It can be seen that $\mathcal{R}$ is homeomorphic to the product of a 4-sphere and an interval $I, S^{4} \times I$; namely, for each fixed value of $p_{1}-q_{1}$ in the interval $[-c, c]$, we see that the equation $H_{2}=h$ determines a 4-sphere

$$
\frac{\lambda}{4}\left(q_{1}+p_{1}\right)^{2}+\frac{v}{2}\left(q_{2}^{2}+p_{2}^{2}\right)+\frac{\omega}{2}\left(q_{3}^{2}+p_{3}^{2}\right)=h+\frac{\lambda}{4}\left(p_{1}-q_{1}\right)^{2} .
$$

The bounding 4-sphere of $\mathcal{R}$ for which $p_{1}-q_{1}=-c$ will be called $n_{1}$, and that where $p_{1}-q_{1}=c, n_{2}$ (see figure 4). We shall call the set of points on each bounding 4-sphere where $q_{1}+p_{1}=0$ the equator, and the sets where $q_{1}+p_{1}>0$ or $q_{1}+p_{1}<0$ will be called the $n$ orth and south hemispheres, respectively.

The linear flow in $\mathcal{R}$. To analyse the flow in $\mathcal{R}$, one considers the projections on the $\left(q_{1}, p_{1}\right)$ plane and $\left(q_{2}, p_{2}\right) \times\left(q_{3}, p_{3}\right)$-space, respectively. In the first case we see the standard picture of an unstable critical point, and in the second, of a centre consisting of two uncoupled harmonic oscillators. Figure 4 schematically illustrates the flow. The coordinate axes of the $\left(q_{1}, p_{1}\right)$ plane have been tilted by $45^{\circ}$ and labelled $\left(p_{1}, q_{1}\right)$ in order to correspond to the direction of the flow in later figures which adopt the NASA convention that the larger primary is to the left of the smaller secondary. With regard to the first projection we see that $\mathcal{R}$ itself projects to a set bounded on two sides by the hyperbola $q_{1} p_{1}=h / \lambda$ (corresponding to $q_{2}^{2}+p_{2}^{2}=q_{3}^{2}+p_{3}^{2}=0$, see (4)) and on two other sides by the line segments $p_{1}-q_{1}= \pm c$, which correspond to the bounding 4-spheres.

Since $q_{1} p_{1}$ is an integral of the equations in $\mathcal{R}$, the projections of orbits in the $\left(q_{1}, p_{1}\right)$ plane move on the branches of the corresponding hyperbolae $q_{1} p_{1}=$ constant, except in the case $q_{1} p_{1}=0$, where $q_{1}=0$ or $p_{1}=0$. If $q_{1} p_{1}>0$, the branches connect the bounding 


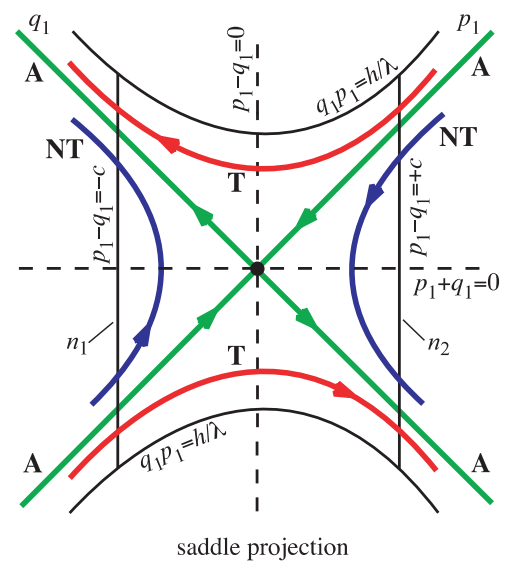

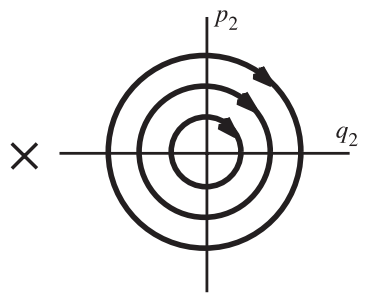

planar oscillations projection

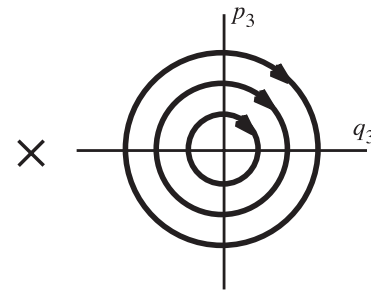

vertical oscillations projection

Figure 4. The flow in the equilibrium region has the form saddle $\times$ centre $\times$ centre. On the left is shown the projection onto the $\left(p_{1}, q_{1}\right)$-plane (note, axes tilted $45^{\circ}$ ). Shown are the bounded orbits (black dot at the centre), the asymptotic orbits (labelled A), two transit orbits (T) and two non-transit orbits (NT).

line segments $p_{1}-q_{1}= \pm c$ and if $q_{1} p_{1}<0$, they have both end points on the same segment. A check of equation (5) shows that the orbits move as indicated by the arrows in figure 4 .

To interpret figure 4 as a flow in $\mathcal{R}$, note that each point in the $\left(q_{1}, p_{1}\right)$-plane projection corresponds to a 3 -sphere $S^{3}$ in $\mathcal{R}$ given by

$$
\frac{v}{2}\left(q_{2}^{2}+p_{2}^{2}\right)+\frac{\omega}{2}\left(q_{3}^{2}+p_{3}^{2}\right)=h-\lambda q_{1} p_{1} .
$$

Of course, for points on the bounding hyperbolic segments $\left(q_{1} p_{1}=h / \lambda\right)$, the 3 -sphere collapses to a point. Thus, the segments of the lines $p_{1}-q_{1}= \pm c$ in the projection correspond to the 4 -spheres bounding $\mathcal{R}$. This is because each corresponds to a 3 -sphere crossed with an interval where the two end 3 -spheres are pinched to a point.

We distinguish nine classes of orbits grouped into the following four categories:

(i) The point $q_{1}=p_{1}=0$ corresponds to an invariant 3-sphere $S_{h}^{3}$ of bounded orbits (periodic and quasi-periodic) in $\mathcal{R}$. This 3 -sphere is given by

$$
\frac{v}{2}\left(q_{2}^{2}+p_{2}^{2}\right)+\frac{\omega}{2}\left(q_{3}^{2}+p_{3}^{2}\right)=h, \quad q_{1}=p_{1}=0
$$

It is an example of a normally hyperbolic invariant manifold (NHIM) (see [42]), where stretching and contraction rates under the linearized dynamics transverse to the 3 -sphere dominate those tangent to the 3 -sphere. This is clear for this example since the dynamics normal to the 3 -sphere are described by the exponential contraction and expansion of the saddle point dynamics. Here, the 3 -sphere acts as a 'big saddle point'. See the black dot at the centre of the $\left(q_{1}, p_{1}\right)$-plane on the left side of figure 4 .

(ii) The four half open segments on the axes, $q_{1} p_{1}=0$, correspond to four cylinders of orbits asymptotic to this invariant 3 -sphere $S_{h}^{3}$ either as time increases $\left(p_{1}=0\right)$ or as time decreases $\left(q_{1}=0\right)$. These are called asymptotic orbits and they form the stable and the unstable manifolds of $S_{h}^{3}$. The stable manifolds, $W_{ \pm}^{s}\left(S_{h}^{3}\right)$, are given by

$$
\frac{v}{2}\left(q_{2}^{2}+p_{2}^{2}\right)+\frac{\omega}{2}\left(q_{3}^{2}+p_{3}^{2}\right)=h, \quad q_{1}=0,
$$



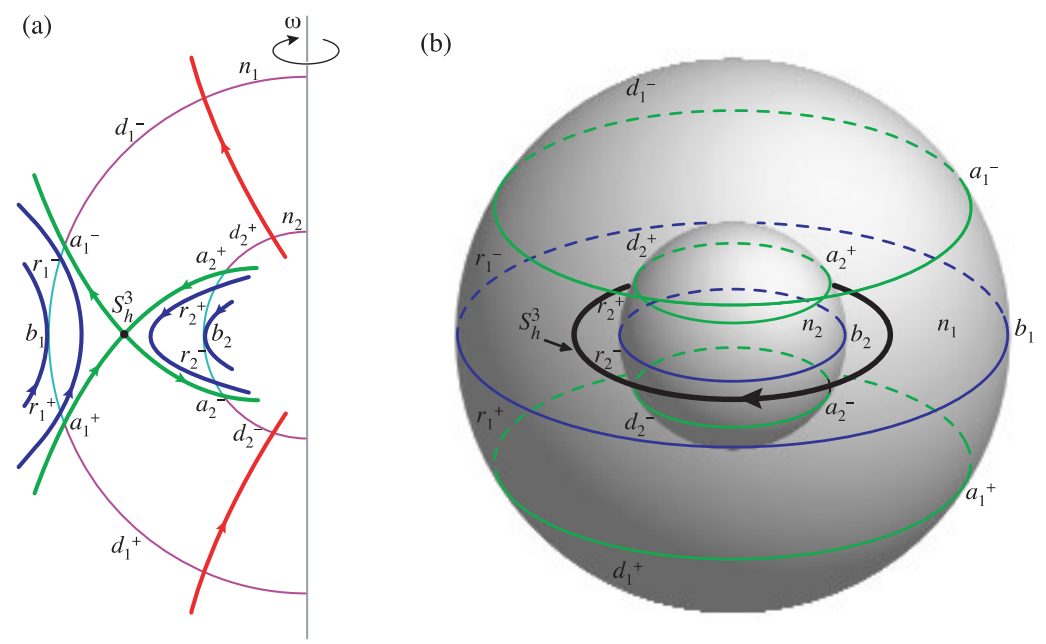

Figure 5. (a) The cross-section of the flow in the $\mathcal{R}$ region of the energy surface. $(b)$ The McGehee representation of the flow in the region $\mathcal{R}$. Adapted from [27].

where $W_{+}^{s}\left(S_{h}^{3}\right)$ (with $p_{1}>0$ ) is the branch going from right to left and $W_{-}^{s}\left(S_{h}^{3}\right)$ (with $\left.p_{1}<0\right)$ is the branch going from left to right. The unstable manifolds, $W_{ \pm}^{u}\left(S_{h}^{3}\right)$, are given by

$$
\frac{v}{2}\left(q_{2}^{2}+p_{2}^{2}\right)+\frac{\omega}{2}\left(q_{3}^{2}+p_{3}^{2}\right)=h, \quad p_{1}=0,
$$

where $W_{+}^{u}\left(S_{h}^{3}\right)$ (with $q_{1}>0$ ) is the branch from right to left and $W_{-}^{u}\left(S_{h}^{3}\right)$ (with $q_{1}<0$ ) is the branch from left to right. See the four orbits labelled A of figure 4.

(iii) The hyperbolic segments determined by $q_{1} p_{1}=$ constant $>0$ correspond to two cylinders of orbits that cross $\mathcal{R}$ from one bounding 4-sphere to the other, meeting both in the same hemisphere; the northern hemisphere if they go from $p_{1}-q_{1}=+c$ to $p_{1}-q_{1}=-c$, and the southern hemisphere in the other case. Since these orbits transit from one region to another, we call them transit orbits. See the two orbits labelled T of figure 4.

(iv) Finally, the hyperbolic segments determined by $q_{1} p_{1}=$ constant $<0$ correspond to two cylinders of orbits in $\mathcal{R}$ each of which runs from one hemisphere to the other hemisphere on the same bounding 4-sphere. Thus, if $q_{1}>0$, the 4-sphere is $n_{1}\left(p_{1}-q_{1}=-c\right)$ and orbits run from the southern hemisphere $\left(q_{1}+p_{1}<0\right)$ to the northern hemisphere $\left(q_{1}+p_{1}>0\right)$ while the converse holds if $q_{1}<0$, where the 4 -sphere is $n_{2}$. Since these orbits return to the same region, we call them non-transit orbits. See the two orbits labelled NT of figure 4.

McGehee representation. McGehee [33] proposed a representation which makes it easier to visualize the region $\mathcal{R}$, providing further geometrical insight into the dynamics. As noted above, $\mathcal{R}$ is a five-dimensional manifold that is homeomorphic to $S^{4} \times I$. It can be represented by a spherical annulus bounded by two 4 -spheres $n_{1}, n_{2}$, as shown in figure 5(b). Figure 5(a) is a cross-section of $\mathcal{R}$. Note that this cross-section is qualitatively the same as the illustration in figure 4. The following classifications of orbits correspond to the previous four categories:

(i) There is an invariant 3 -sphere $S_{h}^{3}$ of bounded orbits in the region $\mathcal{R}$ corresponding to the black dot in the middle of figure $5(a)$. Note that this 3 -sphere is the equator of the central 4-sphere given by $p_{1}-q_{1}=0$. 
(ii) Again let $n_{1}, n_{2}$ be the bounding 4-spheres of region $\mathcal{R}$, and let $n$ denote either $n_{1}$ or $n_{2}$. We can divide $n$ into two hemispheres: $n^{+}$, where the flow enters $\mathcal{R}$, and $n^{-}$, where the flow leaves $\mathcal{R}$. There are four cylinders of orbits asymptotic to the invariant 3-sphere $S_{h}^{3}$. They form the stable and unstable manifolds to the invariant 3-sphere $S_{h}^{3}$. Topologically, both invariant manifolds look like four-dimensional 'tubes' $\left(S^{3} \times \mathbb{R}\right)$ inside a five-dimensional energy manifold. The interior of the stable manifolds $W_{ \pm}^{s}\left(S_{h}^{3}\right)$ and unstable manifolds $W_{ \pm}^{u}\left(S_{h}^{3}\right)$ can be given as follows:

$$
\begin{aligned}
& \operatorname{int}\left(W_{+}^{s}\left(S_{h}^{3}\right)\right)=\left\{\left(q_{1}, p_{1}, q_{2}, p_{2}, q_{3}, p_{3}\right) \in \mathcal{R} \mid p_{1}>q_{1}>0\right\}, \\
& \operatorname{int}\left(W_{-}^{s}\left(S_{h}^{3}\right)\right)=\left\{\left(q_{1}, p_{1}, q_{2}, p_{2}, q_{3}, p_{3}\right) \in \mathcal{R} \mid p_{1}<q_{1}<0\right\}, \\
& \operatorname{int}\left(W_{+}^{u}\left(S_{h}^{3}\right)\right)=\left\{\left(q_{1}, p_{1}, q_{2}, p_{2}, q_{3}, p_{3}\right) \in \mathcal{R} \mid q_{1}>p_{1}>0\right\}, \\
& \operatorname{int}\left(W_{-}^{u}\left(S_{h}^{3}\right)\right)=\left\{\left(q_{1}, p_{1}, q_{2}, p_{2}, q_{3}, p_{3}\right) \in \mathcal{R} \mid q_{1}<p_{1}<0\right\} .
\end{aligned}
$$

The exterior of these invariant manifolds can be given similarly from studying figure $5(a)$.

(iii) Let $a^{+}$and $a^{-}$(where $q_{1}=0$ and $p_{1}=0$, respectively) be the intersections of the stable and unstable manifolds with the bounding sphere $n$. Then, $a^{+}$appears as a 3 -sphere in $n^{+}$, and $a^{-}$appears as a 3 -sphere in $n^{-}$. Consider the two spherical caps on each bounding 4-sphere given by

$$
\begin{aligned}
& d_{1}^{+}=\left\{\left(q_{1}, p_{1}, q_{2}, p_{2}, q_{3}, p_{3}\right) \in \mathcal{R} \mid p_{1}-q_{1}=-c, p_{1}<q_{1}<0\right\}, \\
& d_{1}^{-}=\left\{\left(q_{1}, p_{1}, q_{2}, p_{2}, q_{3}, p_{3}\right) \in \mathcal{R} \mid p_{1}-q_{1}=-c, q_{1}>p_{1}>0\right\}, \\
& d_{2}^{+}=\left\{\left(q_{1}, p_{1}, q_{2}, p_{2}, q_{3}, p_{3}\right) \in \mathcal{R} \mid p_{1}-q_{1}=+c, p_{1}>q_{1}>0\right\}, \\
& d_{2}^{-}=\left\{\left(q_{1}, p_{1}, q_{2}, p_{2}, q_{3}, p_{3}\right) \in \mathcal{R} \mid p_{1}-q_{1}=+c, q_{1}<p_{1}<0\right\} .
\end{aligned}
$$

Since $d_{1}^{+}$is the spherical cap in $n_{1}^{+}$bounded by $a_{1}^{+}$, the transit orbits entering $\mathcal{R}$ on $d_{1}^{+}$ exit on $d_{2}^{-}$of the other bounding sphere. Similarly, since $d_{1}^{-}$is the spherical cap in $n_{1}^{-}$ bounded by $a_{1}^{-}$, the transit orbits leaving on $d_{1}^{-}$have come from $d_{2}^{+}$on the other bounding sphere. Note that all spherical caps where the transit orbits pass through are in the interior of stable and unstable manifold tubes.

(iv) Let $b$ be the intersection $b$ of $n^{+}$and $n^{-}$(where $q_{1}+p_{1}=0$ ). Then, $b$ is a 3-sphere of tangency points. Orbits tangent at this 3 -sphere 'bounce off,' i.e. do not enter $\mathcal{R}$ locally. Moreover, if we let $r^{+}$be a spherical zone, which is bounded by $a^{+}$and $b$, then non-transit orbits entering $\mathcal{R}$ on $r^{+}$exit on the same bounding 4-sphere through $r^{-}$which is bounded by $a^{-}$and $b$. It is easy to show that all the spherical zones where non-transit orbits bounce off are in the exterior of stable and unstable manifold tubes.

Invariant manifolds as separatrices. The key observation here is that the asymptotic orbits form four-dimensional stable and unstable manifold 'tubes' $\left(S^{3} \times \mathbb{R}\right)$ to the invariant 3-sphere $S_{h}^{3}$ in a five-dimensional energy surface and they separate two distinct types of motion: transit orbits and non-transit orbits. The transit orbits, passing from one region to another, are those inside the four-dimensional manifold tube. The non-transit orbits, which bounce back to their region of origin, are those outside the tube.

In fact, it can be shown that for a value of the Jacobi constant just below that of $L_{1}\left(L_{2}\right)$, the nonlinear dynamics in the equilibrium region $\mathcal{R}_{1}\left(\mathcal{R}_{2}\right)$ is qualitatively the same as the linearized picture that we have shown above (see $[1,34,43])$.

For example, the NHIM for the nonlinear system, which corresponds to the 3-sphere (6) for the linearized system is given by

$\mathcal{S}_{h}=\left\{(q, p) \mid \frac{v}{2}\left(q_{2}^{2}+p_{2}^{2} t\right)+\frac{\omega}{2}\left(q_{3}^{2}+p_{3}^{2}\right)+f\left(q_{2}, p_{2}, q_{3}, p_{3}\right)=h, q_{1}=p_{1}=0\right\}$, 
where $f$ is at least of third order. Here, $\left(q_{1}, p_{1}, q_{2}, p_{2}, q_{3}, p_{3}\right)$ are normal form coordinates and are related to the linearized coordinates via a near-identity transformation.

In a small neighbourhood of the equilibrium point, since the higher order terms in $f$ are much smaller than the second-order terms, the 3-sphere for the linear problem becomes a deformed sphere for the nonlinear problem. Moreover, since NHIMs persist under perturbation, this deformed sphere $\mathcal{S}_{h}$ still has stable and unstable manifolds which are given by

$W_{ \pm}^{s}\left(\mathcal{S}_{h}\right)=\left\{(q, p) \mid \frac{v}{2}\left(q_{2}^{2}+p_{2}^{2}\right)+\frac{\omega}{2}\left(q_{3}^{2}+p_{3}^{2}\right)+f\left(q_{2}, p_{2}, q_{3}, p_{3}\right)=h, \quad q_{1}=0\right\}$,
$W_{ \pm}^{u}\left(\mathcal{S}_{h}\right)=\left\{(q, p) \mid \frac{v}{2}\left(q_{2}^{2}+p_{2}^{2}\right)+\frac{\omega}{2}\left(q_{3}^{2}+p_{3}^{2}\right)+f\left(q_{2}, p_{2}, q_{3}, p_{3}\right)=h, \quad p_{1}=0\right\}$.

Note the similarity between the formulae above and those for the linearized problem given by equations (7) and (8).

See the appendix at the end of this paper as well as other references $[15,23,19,43]$ for details. This geometric insight will be used below to guide our numerical explorations in constructing orbits with prescribed itineraries.

\section{Construction of orbits with prescribed itineraries in the planar case}

In this section, we review previous work on the planar case $[27,30]$ which provides the necessary background for the extension of these ideas to the spatial case. In [30], a numerical demonstration is given of a heteroclinic connection between pairs of equal Jacobi constant Lyapunov orbits, one around $L_{1}$, the other around $L_{2}$. This heteroclinic connection augments the homoclinic orbits associated with the $L_{1}$ and $L_{2}$ Lyapunov orbits, which were previously known [33]. Linking these heteroclinic connections and homoclinic orbits leads to dynamical chains.

The dynamics in the neighbourhood of these chains give rise to interesting analytical results. We proved the existence of a large class of interesting orbits near a chain, which a spacecraft can follow in its rapid transition between the inside and outside of a Jovian moon's orbit via a moon encounter. The global collection of these orbits is called a dynamical channel. We proved a theorem which gives the global orbit structure in the neighbourhood of a chain. In simplified form, the theorem essentially says:

For any admissible bi-infinite sequence $\left(\ldots, u_{-1} ; u_{0}, u_{1}, u_{2}, \ldots\right)$ of symbols $\{I, M, X\}$ where $I, M$ and $X$ stand for the interior, moon and exterior regions, respectively, there corresponds an orbit near the chain whose past and future whereabouts with respect to these three regions match those of the given sequence.

For example, consider the Jupiter-Ganymede-spacecraft three-body system. Given the bi-infinite sequence $(\ldots, I ; M, X, M, \ldots)$, there exists an orbit starting in the Ganymede region, which came from the interior region and is going to the exterior region and returning to the Ganymede region.

Moreover, we not only proved the existence of orbits with prescribed itineraries, but developed a systematic procedure for their numerical construction. We will illustrate below the numerical construction of orbits with prescribed finite (but large enough) itineraries in the three-body planet-moon-spacecraft problem. As our example, chosen for simplicity of exposition, we construct a spacecraft orbit with the central block $(M, X ; M, I, M)$.

Example itinerary: $(M, X ; M, I, M)$. For the present numerical construction, we adopt the following convention. The $U_{1}$ and $U_{4}$ Poincare sections will be $(y=0, x<0, \dot{y}<0)$ in the 

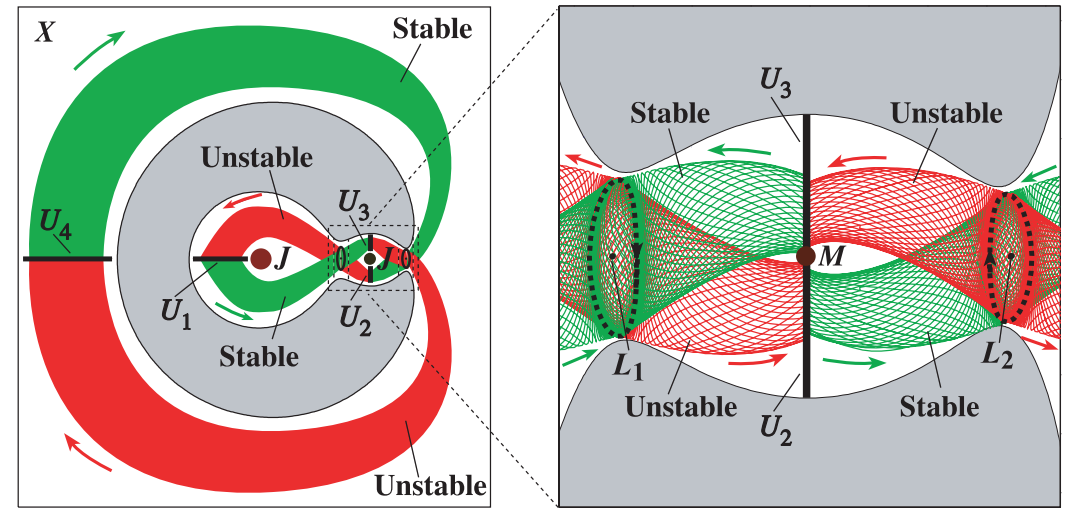

Figure 6. Location of libration point orbit invariant manifold tubes in position space. Stable manifolds are lightly shaded, unstable manifolds are dark. The location of the Poincaré sections $\left(U_{1}, U_{2}, U_{3}\right.$, and $\left.U_{4}\right)$ are also shown.

interior region, and $(y=0, x<-1, \dot{y}>0)$ in the exterior region, respectively. The $U_{2}$ and $U_{3}$ sections will be $(x=1-\mu, y<0, \dot{x}>0)$ and $(x=1-\mu, y>0, \dot{x}<0)$ in the moon region, respectively. See figure 6 for the location of the Poincaré sections relative to the tubes.

Figures $7(a)$ and $(b)$ provide a numerical example of how the invariant manifold tubes separate two types of motion. Consider the $L_{1}$ side. The set of right-to-left transit orbits has the structure $D^{2} \times \mathbb{R}$ (where $D^{2}$ is a two-dimensional disc), with boundary $S^{1} \times \mathbb{R}$. The boundary is made up of $W_{+}^{s}\left(\mathcal{S}_{h}^{1}\right)$ and $W_{+}^{u}\left(\mathcal{S}_{h}^{1}\right)$, where the + means right-to-left, $\mathcal{S}_{h}^{1}$ is the NHIM around $L_{1}$ (a periodic orbit in this case) with energy $h$ and the superscript $i$ denotes $L_{i}$. We pick the initial conditions to approximate $W_{+}^{s}\left(\mathcal{S}_{h}^{1}\right)$ as outlined in [27] and then integrate those initial conditions forward in time until they intersect the Poincaré section $U_{3}$. This is how the upper curve in figure $7(b)$ is generated, which we refer to as the Poincaré cut of $W_{+}^{s}\left(\mathcal{S}_{h}^{1}\right)$. A point inside that curve is an orbit that goes from the moon region to the interior region, so this region can be described by the label $(; M, I)$.

Similarly, a point inside the lower curve of figure $7(b)$, the Poincare cut of $W_{+}^{u}\left(\mathcal{S}_{h}^{2}\right)$ for the same $h$, came from the exterior region into the moon region, and so has the label $(X ; M)$. A point inside the intersection $\Delta_{\mathcal{M}}$ of both curves is an $(X ; M, I)$ orbit, so it makes a transition from the exterior region to the interior region, passing through the moon region. Similarly, by choosing Poincaré sections in the interior and the exterior region, i.e. in the $U_{1}$ and $U_{4}$ plane, we find the intersection region $\Delta_{\mathcal{I}}$ consisting of $(M ; I, M)$ orbits, and $\Delta_{\mathcal{X}}$, which consists of $(M ; X, M)$ orbits.

Flowing the intersection $\Delta_{\mathcal{X}}$ forward to the moon region, it stretches into the strips in figure $7(c)$. These strips are the image of $\Delta_{\mathcal{X}}$ (i.e. $P\left(\Delta_{\mathcal{X}}\right)$ ) under the Poincare map $P$, and thus get the label $(M, X ; M)$. Similarly, flowing the intersection $\Delta_{I}$ backwards to the moon region, it stretches into the strips $P^{-1}\left(\Delta_{\mathcal{I}}\right)$ in figure $7(c)$, and thus has the label $(; M, I, M)$. The intersection of these two types of strips (i.e. $\Delta_{\mathcal{M}} \cap P\left(\Delta_{\mathcal{X}}\right) \cap P^{-1}\left(\Delta_{\mathcal{I}}\right)$ ) consists of the desired $(M, X ; M, I, M)$ orbits. If we take any point inside these intersections and integrate it forwards and backwards, we find the desired orbits (see figure $7(d)$ ).

\section{Extension of results in the planar model to the spatial model}

Since the key step in the planar case is to find the intersection region inside the two Poincaré cuts, a key difficulty is to determine how to extend this technique to the spatial case. Take 

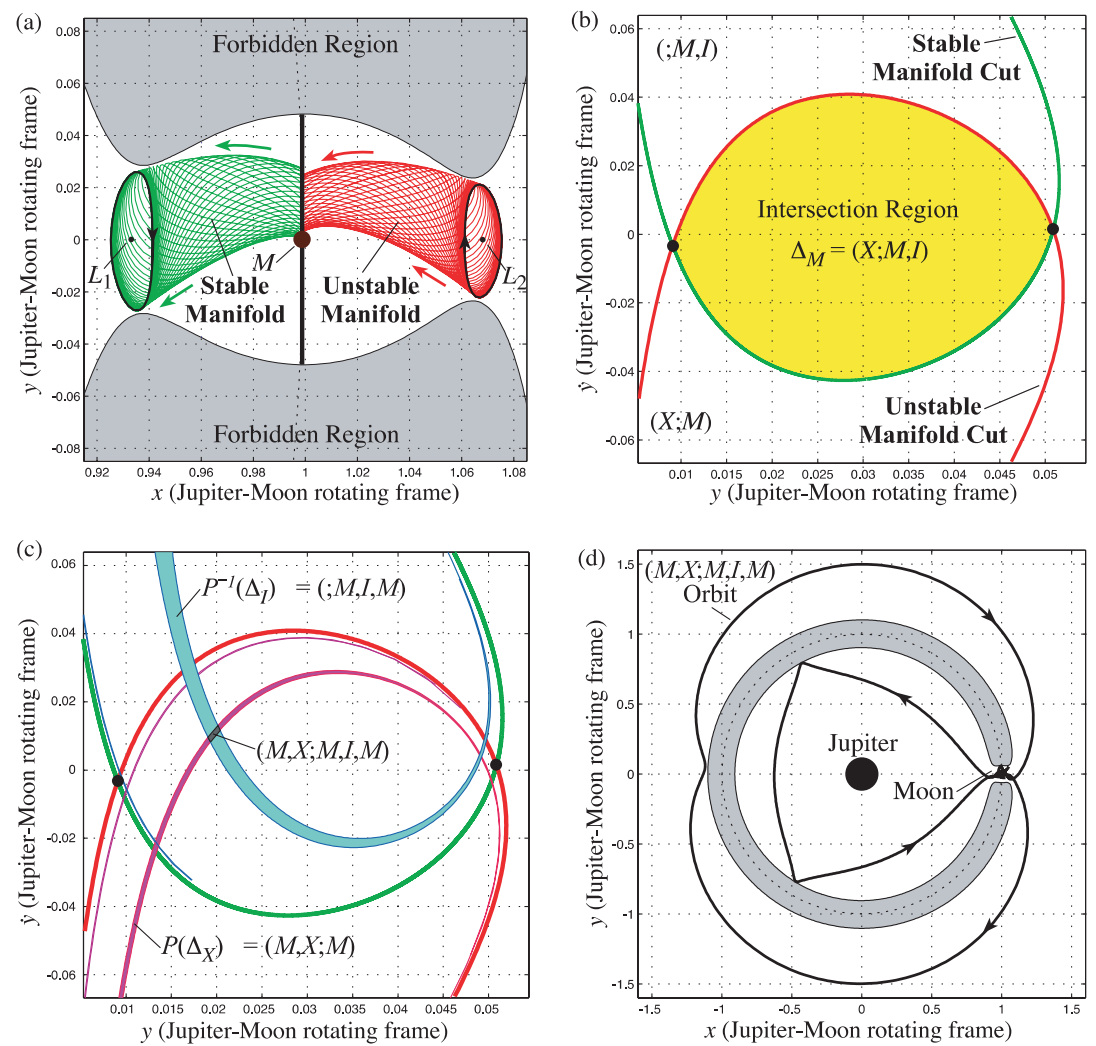

Figure 7. (a) The projection of invariant manifolds $W_{+}^{s}\left(\mathcal{S}_{h}^{1}\right)$ and $W_{+}^{u}\left(\mathcal{S}_{h}^{2}\right)$ in the region $M$ of the position space. $(b)$ A close-up of the intersection region between the Poincare cuts of the invariant manifolds on the $U_{3}$ section $(x=1-\mu, y>0)$. (c) Intersection between image of $\Delta_{X}$ and pre-image of $\Delta_{I}$ labelled $(M, X ; M, I, M) .(d)$ Example orbit passing through $(M, X ; M, I, M)$ region of $(c)$. (Adapted from [27].)

as an example the construction of a transit orbit with the itinerary $(X ; M, I)$ that goes from the exterior region to the interior region of the Jupiter-moon system. Recall that in the spatial case, the unstable manifold 'tube' of the NHIM around $L_{2}$, which separates the transit and non-transit orbits, is topologically $S^{3} \times \mathbb{R}$. For a transversal cut at $x=1-\mu$ (a hyperplane through the moon), the Poincaré cut is a topological 3-sphere $S^{3}$ (in $\mathbb{R}^{4}$ ). It is not obvious how to find the intersection region inside these two Poincare cuts $\left(S^{3}\right)$ since both its projections on the $(y, \dot{y})$-plane and the $(z, \dot{z})$-plane are (two-dimensional) discs $D^{2}$.

However, in constructing an orbit which transits from outside to inside a moon's orbit, suppose that we might also want it to have other characteristics above and beyond this gross behaviour. We may want to have an orbit which has a particular $z$-amplitude when it is near the moon. If we set $z=c, \dot{z}=0$, where $c$ is the desired $z$-amplitude, the problem of finding the intersection region inside two Poincare cuts suddenly becomes tractable. The set of orbits in the Poincaré cut of the unstable manifold with $(z, \dot{z})=(c, 0)$, a point on the $(z, \dot{z})$-plane, projects to a closed curve on the $(y, \dot{y})$-plane. Any point $(z, \dot{z})=(c, 0)$ which is inside this curve on the $(y, \dot{y})$-plane is a $(X ; M)$ orbit which has transited from the exterior region to the moon region passing through the $L_{2}$ equilibrium region.

Similarly, we can apply the same techniques to the Poincaré cut of the stable manifold tube to the NHIM around $L_{1}$ and find all $(M, I)$ orbits with $(z, \dot{z})=(c, 0)$ inside a closed curve in 
the $(y, \dot{y})$-plane. Hence, by using $z$ and $\dot{z}$ as the additional parameters, we can apply similar techniques that we have developed for the planar case in constructing spatial trajectories with the desired itineraries. What follows is a more detailed description.

Finding the Poincaré cuts. We begin with the 15th order normal form expansion near $L_{1}$ and $L_{2}$. See the appendix of this paper and other references $[12,15,23]$ for details. The behaviour of orbits in the coordinate system of that normal form, $\left(q_{1}, p_{1}, q_{2}, p_{2}, q_{3}, p_{3}\right)$, is qualitatively similar to the behaviour of orbits in the linear approximation. This makes the procedure for choosing initial conditions in the $L_{1}$ and $L_{2}$ equilibrium regions rather simple. In particular, based on our knowledge of the structure for the linear system, we can pick initial conditions which produce a close 'shadow' of the stable and unstable manifold 'tubes' $\left(S^{3} \times \mathbb{R}\right)$ associated with the NHIM, also called the central or neutrally stable manifold, in both the $L_{1}$ and $L_{2}$ equilibrium regions. As we restrict to an energy surface with energy $h$, there is only one NHIM per energy surface, denoted by $\mathcal{S}_{h}\left(\simeq S^{3}\right)$.

The initial conditions in $\left(q_{1}, p_{1}, q_{2}, p_{2}, q_{3}, p_{3}\right)$ are picked with the qualitative picture of the linear system in mind. The coordinates $\left(q_{1}, p_{1}\right)$ correspond to the saddle projection, $\left(q_{2}, p_{2}\right)$ correspond to oscillations within the $(x, y)$ plane, and $\left(q_{3}, p_{3}\right)$ correspond to oscillations within the $z$-direction. Also, note that $q_{3}=p_{3}=0(z=\dot{z}=0)$ corresponds to an invariant manifold of the system, i.e. the planar system is an invariant manifold of the three degrees of freedom system.

The initial conditions to approximate the stable and unstable manifolds $\left(W_{ \pm}^{s}\left(\mathcal{S}_{h}\right), W_{ \pm}^{u}\left(\mathcal{S}_{h}\right)\right)$ are picked via the following procedure. Note that we can be assured that we are obtaining a roughly complete approximation of points along a slice of $W_{ \pm}^{s}\left(\mathcal{S}_{h}\right)$ and $W_{ \pm}^{u}\left(\mathcal{S}_{h}\right)$ since such a slice is compact, having the structure $S^{3}$. Also, we know the picture roughly from the linear case.

(i) We fix $q_{1}=p_{1}= \pm \epsilon$, where $\epsilon$ is small. This ensures that almost all of the initial conditions will be for orbits which are transit orbits from one side of the equilibrium region to the other. Specifically ' + ' corresponds to right-to-left transit orbits and '-' corresponds to left-to-right transit orbits. We choose $\epsilon$ small so that the initial conditions are near the NHIM $\mathcal{S}_{h}$ (at $q_{1}=p_{1}=0$ ) and will, therefore, integrate forwards and backwards to be near the unstable and stable manifolds of $\mathcal{S}_{h}$, respectively. We choose $\epsilon$ to not be too small, or the integrated orbits will take too long to leave the vicinity of $\mathcal{S}_{h}$.

(ii) Beginning with $r_{v}=0$, and increasing incrementally to some maximum $r_{v}=r_{v}^{\max }$, we look for initial conditions with $q_{3}^{2}+p_{3}^{2}=r_{v}^{2}$, i.e. along circles in the $z$ oscillation canonical plane. It is reasonable to look along circles centred on the origin $\left(q_{3}, p_{3}\right)=(0,0)$ on this canonical plane since the motion is simple harmonic in the linear case and the origin corresponds to an invariant manifold.

(iii) For each point along the circle, we look for the point on the energy surface in the $\left(q_{2}, p_{2}\right)$ plane, i.e. the $(x, y)$ oscillation canonical plane. Note, our procedure can tell us if such a point exists, and clearly if no point exists, it will not be used as an initial condition.

After picking the initial conditions in $\left(q_{1}, p_{1}, q_{2}, p_{2}, q_{3}, p_{3}\right)$ coordinates, we transform to the conventional CR3BP coordinates $(x, y, z, \dot{x}, \dot{y}, \dot{z})$ and integrate under the full equations of motion. The integration proceeds until some Poincaré section stopping condition is reached, for example $x=1-\mu$. We can then use further analysis on the Poincaré section, described later.

Example itinerary: $(X ; M, I)$. As an example, suppose we want a transition orbit going from outside to inside the moon's orbit in the Jupiter-moon system. We, therefore, want right-to-left 

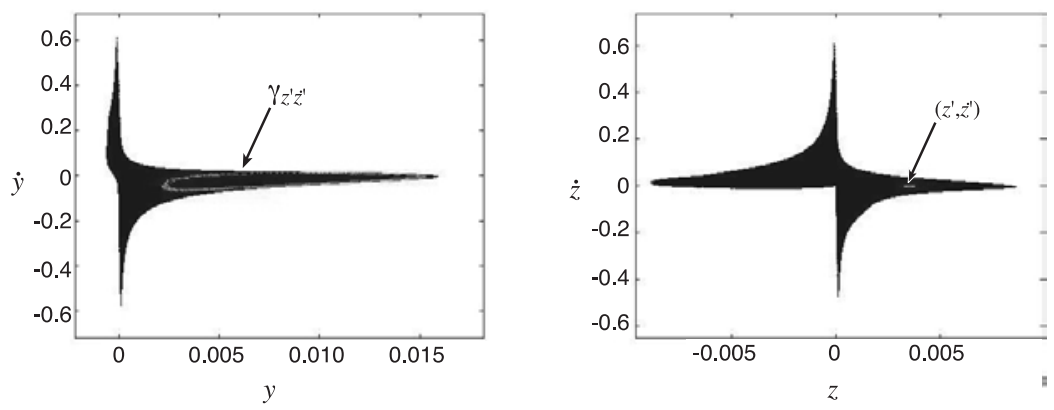

Figure 8. Shown in black are the projections of the three-dimensional object $\mathcal{C}_{1}^{+u 2}$ on the $(y, \dot{y})$ plane (left) and the $(z, \dot{z})$-plane (right), the intersection of $W_{+}^{u}\left(\mathcal{S}_{h}^{2}\right)$ with the Poincare section $x=1-\mu$. The set of points in the $(y, \dot{y})$ projection which approximate a curve, $\gamma_{z^{\prime} \dot{z}^{\prime}}$, all have $(z, \dot{z})$ values within the small box shown in the $(z, \dot{z})$ projection (which appears as a thin strip), centred on $\left(z^{\prime}, \dot{z}^{\prime}\right)$. This example is computed in the Jupiter-Europa system for $C=3.0028$.

transit orbits in both the $L_{1}$ and $L_{2}$ equilibrium regions. Consider the $L_{2}$ side. The set of rightto-left transit orbits has the structure $D^{4} \times \mathbb{R}$ (where $D^{4}$ is a four-dimensional disc), with boundary $S^{3} \times \mathbb{R}$. The boundary is made up of $W_{+}^{s}\left(\mathcal{S}_{h}^{2}\right)$ in the $X$ region and $W_{+}^{u}\left(\mathcal{S}_{h}^{2}\right)$ in the $M$ region, where the + means right-to-left, $\mathcal{S}_{h}^{2}$ is the NHIM around $L_{2}$ with energy $h$, and the superscript $i$ denotes $L_{i}$. In practice, we pick the initial conditions to approximate $W_{+}^{u}\left(\mathcal{S}_{h}^{2}\right)$ as outlined above and then integrate those initial conditions forward in time until they intersect the Poincare section at $x=1-\mu$, a hyperplane passing through the centre of the moon.

Since the Hamiltonian energy $h$ (Jacobi constant) is fixed, the set of all values $\mathcal{C}=$ $\{(y, \dot{y}, z, \dot{z})\}$ obtained at the Poincaré section, characterize the branch of the manifold of all libration point orbits around the selected equilibrium point for the particular section. Let us denote the set as $\mathcal{C}_{i}^{+m j}$, where + denotes the right-to-left branch of the stable $(m=s)$ or unstable $\left(m=u\right.$ ) manifold of the $L_{j}, j=1,2$ libration point orbits at the $i$ th intersection with $x=1-\mu$. We will look at the first intersection, so we have $\mathcal{C}_{1}^{+u 2}$.

The object $\mathcal{C}_{1}^{+u 2}$ is three-dimensional $\left(\simeq S^{3}\right)$ in the four-dimensional space of variables $(y, \dot{y}, z, \dot{z})$. For the Jupiter-Europa system, we show $\mathcal{C}_{1}^{+u 2}$ for the Jacobi constant $C=3.0028$ in figure 8 .

Thus, we suspect that if we pick almost any point $\left(z^{\prime}, \dot{z}^{\prime}\right)$ in the $z \dot{z}$ projection, it corresponds to a closed loop $\gamma_{z^{\prime} \dot{z}^{\prime}}\left(\simeq S^{1}\right)$ in the $y \dot{y}$ projection (see figure 8). Any initial condition $\left(y^{\prime}, \dot{y}^{\prime}, z^{\prime}, \dot{z}^{\prime}\right)$, where $\left(y^{\prime}, \dot{y}^{\prime}\right) \in \gamma_{z^{\prime} \dot{z}^{\prime}}$, will be on $W_{+}^{u}\left(\mathcal{S}_{h}^{2}\right)$, and will wind onto a libration point orbit when integrated backwards in time. Thus, $\gamma_{z^{\prime} \dot{z}^{\prime}}$ defines the boundary of right-to-left transit orbits with $(z, \dot{z})=\left(z^{\prime}, \dot{z}^{\prime}\right)$. If we choose $\left(y^{\prime}, \dot{y}^{\prime}\right) \in \operatorname{int}\left(\gamma_{z^{\prime} \dot{z}^{\prime}}\right)$, where $\operatorname{int}\left(\gamma_{z^{\prime} \dot{z}^{\prime}}\right)$ is the region in the $y \dot{y}$ projection enclosed by $\gamma_{z^{\prime} \dot{z}^{\prime}}$, then the initial condition $\left(y^{\prime}, \dot{y}^{\prime}, z^{\prime}, \dot{z}^{\prime}\right)$ will correspond to a right-to-left transit orbit, which will pass through the $L_{2}$ equilibrium region, from the moon region to outside the moon's orbit, when integrated backwards in time.

Similarly, on the $L_{1}$ side, we pick the initial conditions to approximate $W_{+}^{s}\left(\mathcal{S}_{h}^{1}\right)$ as outlined above and then integrate those initial conditions backwards in time until they intersect the Poincaré section at $x=1-\mu$, obtaining $\mathcal{C}_{1}^{+s 1}$. We can do a similar construction regarding transit orbits, etc. To distinguish closed loops $\gamma_{z^{\prime} \dot{z}^{\prime}}$ from $L_{1}$ or $L_{2}$, let us call a loop $\gamma_{z^{\prime} \dot{z}^{\prime}}^{j}$ if it is from $L_{j}, j=1,2$.

To find initial conditions for transition orbits which go from outside the moon's orbit to inside the moon's orbit with respect to Jupiter, i.e. orbits which are right-to-left transit orbits in both the $L_{1}$ and $L_{2}$ equilibrium regions, we need to look at the intersections of the interiors of $\mathcal{C}_{1}^{+u 2}$ and $\mathcal{C}_{1}^{+s 1}$. Figure 9 shows the $(y, \dot{y})$-plane and $(z, \dot{z})$-plane projections of these objects. 

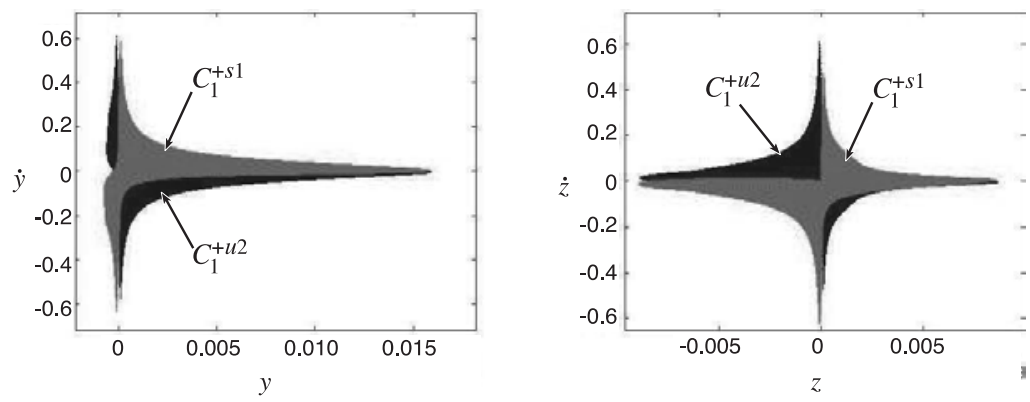

Figure 9. The $(y, \dot{y})$ (left) and $(z, \dot{z})$ (right) projections of the three-dimensional objects $\mathcal{C}_{1}^{+u 2}$ and $\mathcal{C}_{1}^{+s 1}$. This example is computed in the Jupiter-Europa system for $C=3.0028$.

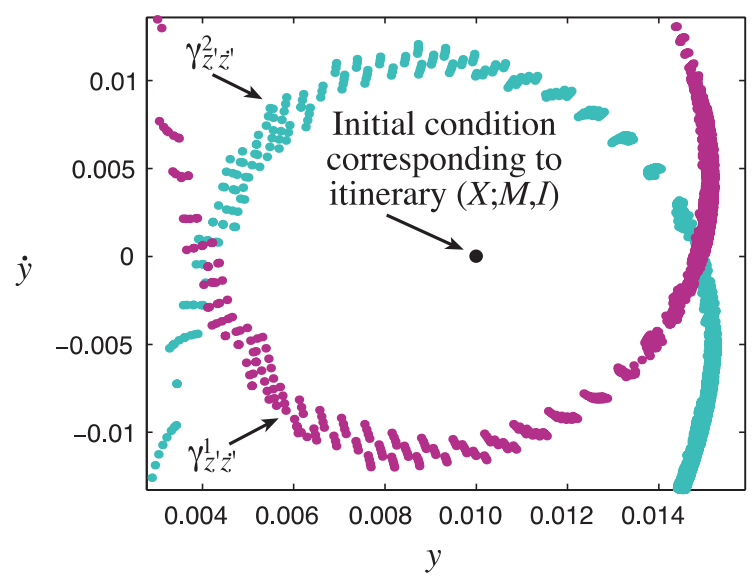

Figure 10. On the $(y, \dot{y})$-plane are shown the points that approximate $\gamma_{z^{\prime} z^{\prime}}^{2}$ and $\gamma_{z^{\prime} \dot{z}^{\prime}}^{1}$, the boundaries of $\operatorname{int}\left(\gamma_{z^{\prime} \dot{z}^{\prime}}^{2}\right)$ and $\operatorname{int}\left(\gamma_{z^{\prime} \dot{z}^{\prime}}^{1}\right)$, respectively, where $\left(z^{\prime}, \dot{z}^{\prime}\right)=(0.0035,0)$. Note the lemon shaped region of intersection, $\operatorname{int}\left(\gamma_{z^{\prime} z^{\prime}}^{1}\right) \cap \operatorname{int}\left(\gamma_{z^{\prime} z^{\prime}}^{2}\right)$, in which all orbits have the itinerary $(X ; M, I)$. The appearance is similar to figure $7(b)$. The point shown within $\operatorname{int}\left(\gamma_{z^{\prime} \dot{z}^{\prime}}^{1}\right) \cap \operatorname{int}\left(\gamma_{z^{\prime} \dot{z}^{\prime}}^{2}\right)$ is the initial condition for the orbit shown in figure 11 .

To find such initial conditions we first look for intersections in the $z \dot{z}$ projection. Consider the projection $\pi_{z \dot{z}}: \mathbb{R}^{4} \rightarrow \mathbb{R}^{2}$ given by $(y, \dot{y}, z, \dot{z}) \mapsto(z, \dot{z})$. Consider a point $\left(y^{\prime}, \dot{y}^{\prime}, z^{\prime}, \dot{z}^{\prime}\right) \in \pi_{z \dot{z}}\left(\mathcal{C}_{1}^{+u 2}\right) \cap \pi_{z \dot{z}}\left(\mathcal{C}_{1}^{+s 1}\right) \neq \varnothing$, i.e. a point $\left(y^{\prime}, \dot{y}^{\prime}, z^{\prime}, \dot{z}^{\prime}\right)$ where $\left(z^{\prime}, \dot{z}^{\prime}\right)$ is in the intersection of the $z \dot{z}$ projections of $\mathcal{C}_{1}^{+u 2}$ and $\mathcal{C}_{1}^{+s 1}$. Transit orbits from outside to inside the moon's orbit are such that $\left(y^{\prime}, \dot{y}^{\prime}, z^{\prime}, \dot{z}^{\prime}\right) \in \operatorname{int}\left(\gamma_{z^{\prime} \dot{z}^{\prime}}^{1}\right) \cap \operatorname{int}\left(\gamma_{z^{\prime} \dot{\prime}^{\prime}}^{2}\right)$. If $\operatorname{int}\left(\gamma_{z^{\prime} \dot{z}^{\prime}}^{1}\right) \cap \operatorname{int}\left(\gamma_{z^{\prime} \dot{z}^{\prime}}^{2}\right)=\varnothing$, then no transition exists for that value of $\left(z^{\prime}, \dot{z}^{\prime}\right)$. But, numerically, we find that there are values of $\left(z^{\prime}, \dot{z}^{\prime}\right)$ such that $\operatorname{int}\left(\gamma_{z^{\prime} z^{\prime}}^{1}\right) \cap \operatorname{int}\left(\gamma_{z^{\prime} \dot{z}^{\prime}}^{2}\right) \neq \varnothing$ (see figures 9 and 10). The initial condition labelled in figure 10 is integrated forwards and backwards to construct the $(X, M, I)$ transit orbit shown in figure 11.

In essence we are doing a search for transit orbits by looking at a two parameter set of intersections of the interiors of closed curves, $\gamma_{z \dot{z}}^{1}$ and $\gamma_{z \dot{z}}^{2}$ in the $y \dot{y}$ projection, where our two parameters are given by $(z, \dot{z})$. Furthermore, we can reduce this to a one parameter family of intersections by restricting to $\dot{z}=0$. This is a convenient choice since it implies that the orbit is at a critical point (often a maximum or minimum in $z$ when it reaches the surface $x=1-\mu$.)

We are approximating the three-dimensional surface $\mathcal{C}$ by a scattering of points (about a million for the computations in this paper), we must look not at points $(z, \dot{z})$, but at small 

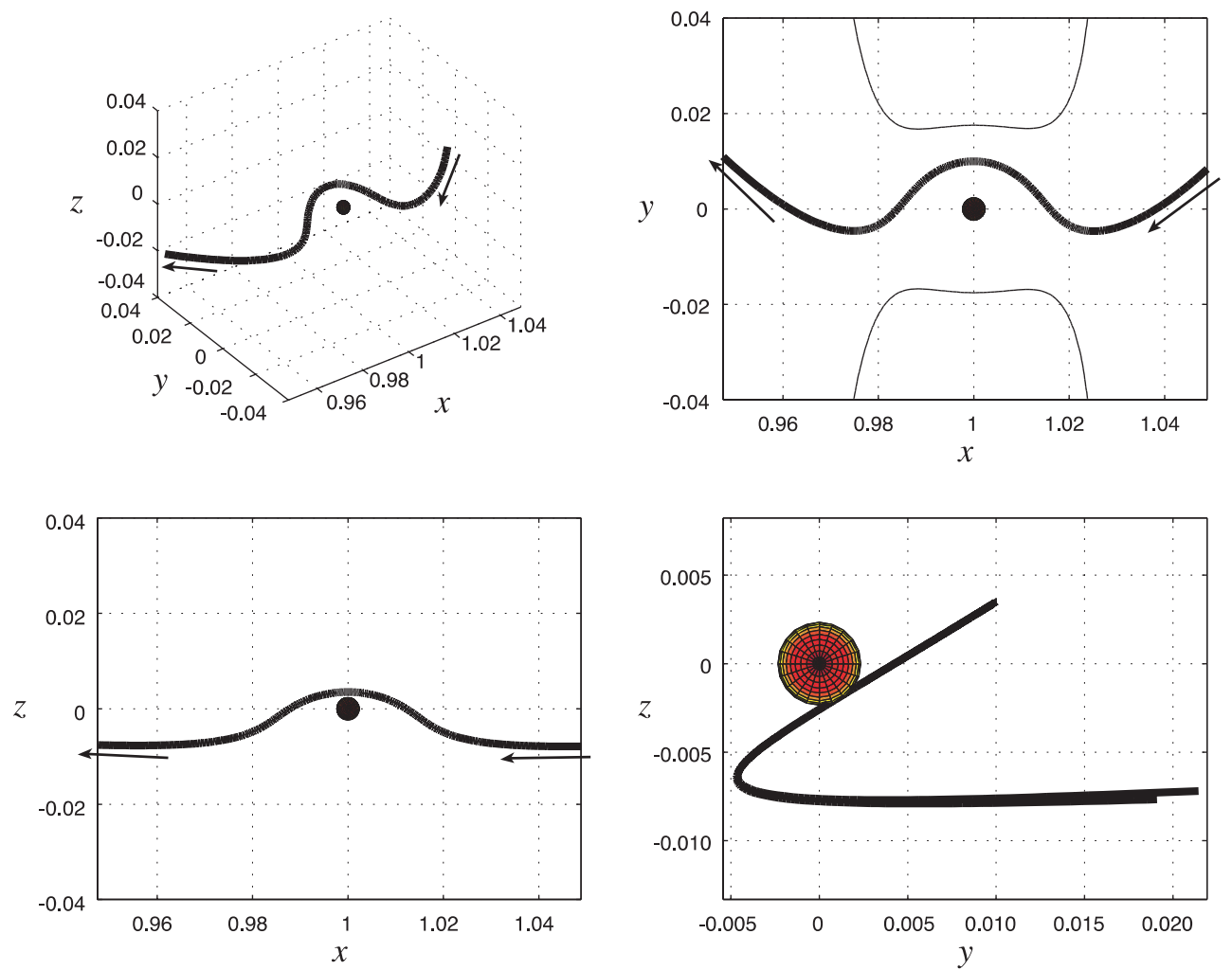

Figure 11. The $(X, M, I)$ transit orbit corresponding to the initial condition in figure 10 . The orbit is shown in a three-dimensional view and in the three orthographic projections. Europa is shown to scale. The upper right plot includes the $z=0$ section of the zero velocity surface (compare with figure $3(b))$.

boxes $(z \pm \delta z, \dot{z} \pm \delta \dot{z})$ where $\delta z$ and $\delta \dot{z}$ are small. Since our box in the $z \dot{z}$ projection has a finite size, the points in the $y \dot{y}$ projection corresponding to the points in the box will not all fall on a closed curve, but along a slightly broadened curve, a strip, as seen in figure 10 . A continuation method could be implemented to find the curves $\gamma_{z \dot{z}}^{j}$ belonging to points $(z, \dot{z})$ in the $z \dot{z}$ projection, but the much easier method of scattering points in the small boxes has been enough for our purposes. We will still refer to the collection of such points as $\gamma_{z \dot{z}}^{j}$.

\section{From Ganymede to a high inclination Europa orbit}

Petit Grand Tour. We now apply the techniques we have developed to the construction of a fully three-dimensional Petit Grand Tour of the Jovian moons, extending an earlier planar result [27]. We outline here how one systematically constructs a spacecraft tour which begins beyond Ganymede in orbit around Jupiter, makes a close flyby of Ganymede, and finally reaches a high inclination orbit around Europa, consuming less fuel than is possible from standard two-body methods.

Our approach involves the following three key ideas:

(i) Treat the solution of the spacecraft's motion in the Jupiter-Ganymede-Europa-spacecraft four-body problem as two patched circular restricted three-body solutions, the JupiterGanymede-spacecraft and Jupiter-Europa-spacecraft systems. 


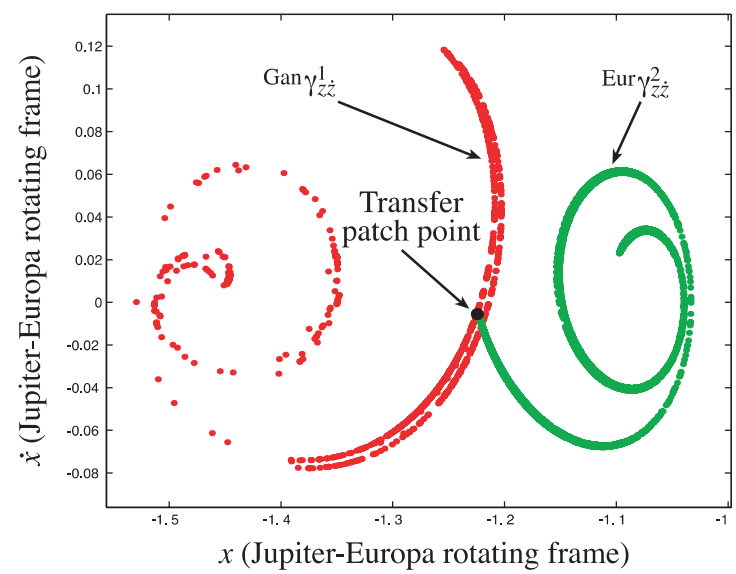

Figure 12. The curves Gan $\gamma_{z \dot{z}}^{1}$ and ${ }^{\text {Eur }} \gamma_{z \dot{z}}^{2}$ are shown, the intersections of Gan $W_{+}^{u}\left(\mathcal{S}^{1}\right)$ and ${ }^{\text {Eur }} W_{+}^{s}\left(\mathcal{S}^{2}\right)$ with the Poincare section $U_{1}$ in the Jupiter-Europa rotating frame, respectively. Note the small region of intersection, int $\left({ }^{\operatorname{Gan}} \gamma_{z \dot{z}}^{1}\right) \cap \operatorname{int}\left({ }^{\text {Eur }} \gamma_{z \dot{z}}^{2}\right)$, where the patch point is labelled.

(ii) Use the stable and unstable manifolds of the NHIMs about the Jupiter-Ganymede $L_{1}$ and $L_{2}$ to find an uncontrolled trajectory from a Jovicentric orbit beyond Ganymede to a temporary capture around Ganymede, which subsequently leaves Ganymede's vicinity onto a Jovicentric orbit interior to Ganymede's orbit.

(iii) Use the stable manifold of the NHIM around the Jupiter-Europa $L_{2}$ to find an uncontrolled trajectory from a Jovicentric orbit between Ganymede and Europa to a temporary capture around Europa. Once the spacecraft is temporarily captured around Europa, a propulsion manoeuvre can be performed when its trajectory is close to Europa ( $100 \mathrm{~km}$ altitude), taking it into a high inclination orbit about the moon. Furthermore, a propulsion manoeuvre will be needed when transferring from the Jupiter-Ganymede portion of the trajectory to the Jupiter-Europa portion, since the respective transport tubes exist at different energies.

Ganymede to Europa transfer mechanism. The construction begins with the patch point, where we connect the Jupiter-Ganymede and Jupiter-Europa portions, and works forwards and backwards in time towards each moon's vicinity. The construction is done mainly in the Jupiter-Europa rotating frame using a Poincaré section. After selecting appropriate energies in each three-body system, respectively, the stable and unstable manifolds of each system's NHIMs are computed. Let ${ }^{\mathrm{Gan}} W_{+}^{u}\left(\mathcal{S}^{1}\right)$ denote the unstable manifold of Ganymede's $L_{1}$ NHIM and Eur $W_{+}^{s}\left(\mathcal{S}^{2}\right)$ denote the stable manifold of Europa's $L_{2}$ NHIM. We look at the intersection of ${ }^{\mathrm{Gan}} W_{+}^{u}\left(\mathcal{S}^{1}\right)$ and ${ }^{\mathrm{Eur}} W_{+}^{s}\left(\mathcal{S}^{2}\right)$ with a common Poincaré section, the surface $U_{1}$ in the Jupiter-Europa rotating frame, defined earlier (see figure 12).

Note that we have the freedom to choose where the Poincare section is with respect to Ganymede, which determines the relative phases of Europa and Ganymede at the patch point. For simplicity, we select the $U_{1}$ surface in the Jupiter-Ganymede rotating frame to coincide with the $U_{1}$ surface in the Jupiter-Europa rotating frame at the patch point. Figure 12 shows the curves ${ }^{\mathrm{Gan}} \gamma_{z \dot{z}}^{1}$ and ${ }^{\mathrm{Eur}} \gamma_{z \dot{z}}^{2}$ on the $(x, \dot{x})$-plane in the Jupiter-Europa rotating frame for all orbits in the Poincare section with points $(z, \dot{z})$ within $(0.0160 \pm 0.0008, \pm 0.0008)$. The size of this range is about $1000 \mathrm{~km}$ in $z$ position and $20 \mathrm{~m} \mathrm{~s}^{-1}$ in $z$ velocity.

From figure 12, an intersection region on the $x \dot{x}$-projection is seen. We pick a point within this intersection region, but with two differing $y$ velocities; one corresponding to 
${ }^{\mathrm{Gan}} W_{+}^{u}\left(\mathcal{S}^{1}\right)$, the tube of transit orbits coming from Ganymede, and the other corresponding to ${ }^{\text {Eur }} W_{+}^{s}\left(\mathcal{S}^{2}\right)$, the orbits heading towards Europa. The discrepancy between these two $y$ velocities is the $\Delta V$ necessary for a propulsive manoeuvre to transfer between the two tubes of transit orbits, which exist at different energies.

Four-body system approximated by patched PCR3BP. In order to determine the transfer $\Delta V$, we compute the transfer trajectory in the full four-body system, taking into account the gravitational attraction of all three massive bodies on the spacecraft. We use the dynamical channel intersection region in the patched three-body model as an initial guess which we adjust finely to obtain a true four-body bi-circular model trajectory (see [30] for more details).

Figure 2 is the final end-to-end trajectory. A $\Delta V$ of $1214 \mathrm{~m} \mathrm{~s}^{-1}$ is required at the location marked. We note that a traditional Hohmann (patched two-body) transfer from Ganymede to Europa requires a $\Delta V$ of $2822 \mathrm{~m} \mathrm{~s}^{-1}$. Our value is only $43 \%$ of the Hohmann value, which is a substantial savings of on-board fuel. The transfer flight time is about 25 days, well within conceivable mission constraints. This trajectory begins on a Jovicentric orbit beyond Ganymede, performs one loop around Ganymede, achieving a close approach of $100 \mathrm{~km}$ above the moon's surface. After the transfer between the two moons, a final additional manoeuvre of $446 \mathrm{~m} \mathrm{~s}^{-1}$ is necessary to enter a high inclination $\left(48.6^{\circ}\right)$ circular orbit around Europa at an altitude of $100 \mathrm{~km}$. Thus, the total $\Delta V$ for the trajectory is $1660 \mathrm{~m} \mathrm{~s}^{-1}$, still substantially lower than the Hohmann transfer value.

\section{Libration orbits around collinear points in the Sun-Earth system}

Besides stable and unstable manifold tubes, centre manifolds of the collinear libration points have played a very important role in space mission design for a long time. Since 1978, when NASA launched the ISEE-3 spacecraft [7,36], Lissajous and halo type trajectories around the collinear libration points of the Sun-Earth system have been considered in the trajectory design of many space missions.

It is well known that halo orbits bifurcate from the planar Lyapunov orbits when the energy reaches a certain level beyond the energy at the corresponding libration point $\left(L_{1}\right.$ or $L_{2}$ ). Hence, there is a need to study the dynamics around an extended neighbourhood of these points in order that more complex missions can be envisaged. In what follows, all computations are done using the CR3BP mass parameter of the Sun-Earth system, where $\mu=3.040423398444176 \times 10^{-6}$.

As has been shown in equation (10) of the previous section, and will be further elaborated in the appendix, the orbits in the centre manifold can be obtained by setting $q_{1}=p_{1}=0$ in the initial conditions of the normal form coordinates $\left(q_{1}, p_{1}, q_{2}, p_{2}, q_{3}, p_{3}\right)$. If we consider only orbits of the same Hamiltonian energy, three free variables remain. Moreover, by looking at the orbits when they cross a surface of section, all the libration orbits with a selected energy value can be obtained from only two variables in the initial conditions. For instance, the initial conditions can be chosen by selecting arbitrary values for $q_{2}$ and $p_{2}$, with $q_{3}=0$ as the surface of section, and finally computing $p_{3}$ in order to be in the selected level of Hamiltonian energy. The propagation of this initial condition, looking when and where it crosses and recrosses the surface of section, gives what are called the images of the Poincare map in the variables $\left(q_{2}, p_{2}\right)$ on $q_{3}=0$.

However, we want to see the orbits in CR3BP coordinates. For this purpose, we can pick the initial conditions as before, transfer to the conventional CR3BP coordinates, integrate under the full equations of motion, and look at the Poincaré map of the orbit when it crosses the 

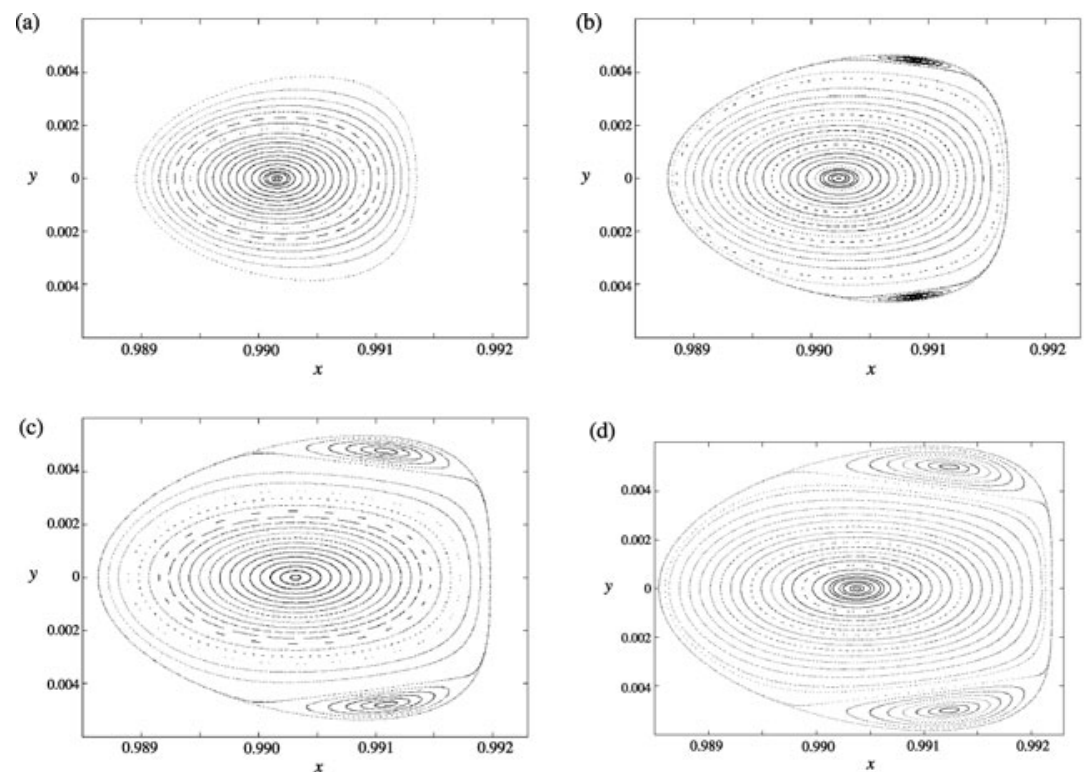

Figure 13. Poincare maps of the orbits in the central manifold of $L_{1}$ for the following decreasing values of the Jacobi constant $C$ : (a) 3.000 85, (b) 3.000 826459 04328 , (c) 3.000 80291513364 , and $(d) 3.00078515837634$. The Poincare section is defined by the plane $z=0$ and is plotted in the position coordinates $(x, y)$.

plane $z=0$ in CR3BP coordinates. The structure of orbits can be clearly seen by plotting their $(x, y)$ coordinates on the section. We note that due to the linear part of the CR3BP equations of motion around the collinear equilibrium points, $z=0$ is a surface of section for all the libration orbits in a neighbourhood of the equilibrium point, except for the planar ones (the ones having $z=\dot{z}=0$ ) which are contained in the $z=0$ plane.

This is the procedure that we have used to obtain figures 13 and 14 , where the libration orbits around $L_{1}$ and $L_{2}$ are displayed for certain values of the Jacobi constant, which give qualitatively different pictures.

We note that for each value of the Jacobi constant $C$, we have a bounded region in the Poincare section. The boundary of the plot is a planar Lyapunov orbit of the selected energy contained in the surface of section. It is the only orbit contained in the $(x, y)$ plane and it is essentially related to the frequency $v$ of $\mathrm{H}_{2}$. The fixed point, in the central part of the figures, corresponds to an almost vertical periodic orbit, essentially related to the frequency $\omega$ of $H_{2}$. Surrounding the central fixed point, we have invariant curves corresponding to Lissajous orbits. The motion in this region is essentially quasi-periodic (except for very small chaotic zones that cannot be seen in the pictures).

Depending on the value of the Jacobi constant, there appear two fixed points closer to the boundaries of the plot. These fixed points correspond to the well-known periodic 'halo' orbits of class I and class II [7]. Surrounding the halo orbit fixed points, we have invariant curves related to quasi-periodic motions. They are Lissajous orbits that we call quasi-halo orbits. See [18] for a study of these orbits.

Finally, in the transition zone from central Lissajous to quasi-halo orbits we find homoclinic points to the planar Lyapunov orbit. We note that at this level of energy the planar Lyapunov orbit is unstable even in the centre manifold. This fact can also be seen compactifying the plot of the Poincaré section in a sphere by means of identifying the Lyapunov orbit at a point. 
(a)
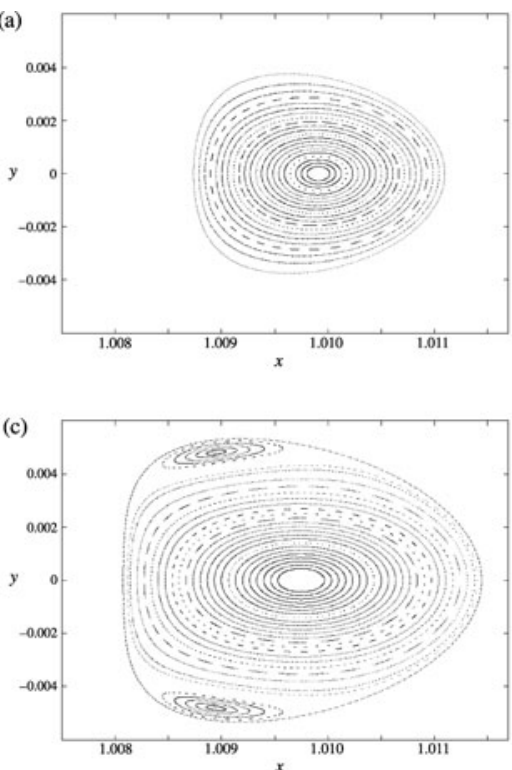

(b)

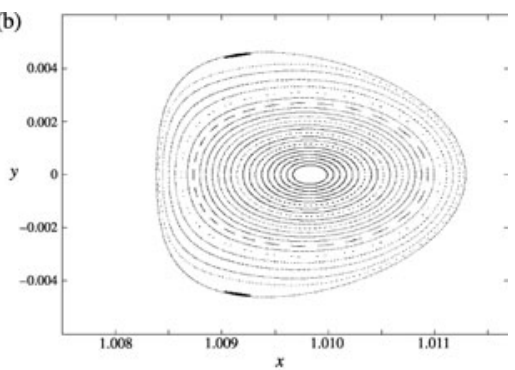

(d)

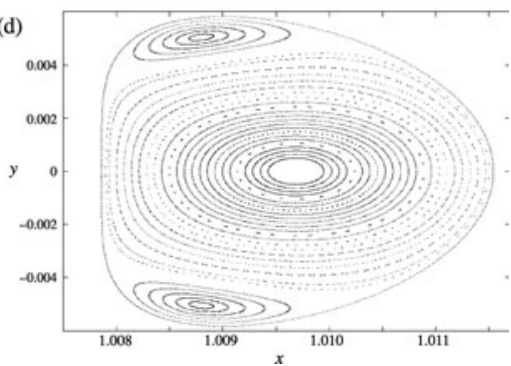

Figure 14. Poincaré maps of the orbits in the central manifold of $L_{2}$ for the following decreasing values of the Jacobi constant $C$ : (a) 3.000 85, (b) 3.000 826459043 28, (c) 3.000 80291513364 , and $(d) 3.00078515837634$. The Poincaré section is defined by the plane $z=0$ and is plotted in the position coordinates $(x, y)$.

The point is a saddle. Inside the centre manifold the planar Lyapunov orbit has an unstable and a stable manifold which are non-planar. Generically, we expect that they do not coincide but intersect transversally, although with a very small angle, giving homoclinic iterates as well as very narrow stochastic zones associated that cannot be seen in the plot. This paper deals with situations that are far from perturbative situations in which (hetero)-homoclinic connections are known, and so analytical techniques of perturbative separatrix splitting are probably difficult to apply and we have not attempted to proceed in that way. For more information on related phenomena, see $[4,5]$.

The planar and vertical Lyapunov, Lissajous, halo, and quasi-halo family of orbits can be computed using ad hoc algebraic manipulators, based on Lindstedt-Poincaré procedures. In this way one obtains their expansions in CR3BP coordinates. See [10, 17, 18, 23] for more details and pictures of the orbits.

\section{Zero cost transfer orbits between libration orbits in the Sun-Earth system}

Besides providing a full description of different kinds of libration motions in a large vicinity of these points, we have also shown the existence of heteroclinic connections between pairs of such libration orbits, one around the libration point $L_{1}$ and the other around $L_{2}$. Since these connections are asymptotic orbits, no manoeuvre is needed to perform the transfer from one libration orbit to the other. Knowledge of these orbits can be very useful in the design of missions such as Genesis [21], and may provide the backbone for other interesting orbits in the future.

In order to find some heteroclinic trajectories connecting libration orbits around $L_{1}$ with those around $L_{2}$, we have to match an orbit of the unstable manifold of a libration orbit around 
(a)

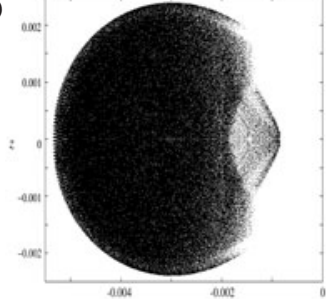

(a1)

(b)

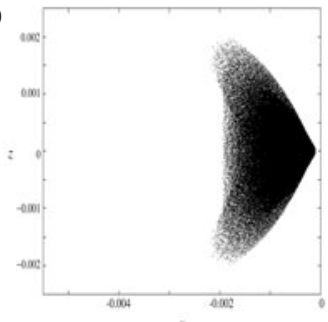

(b1)

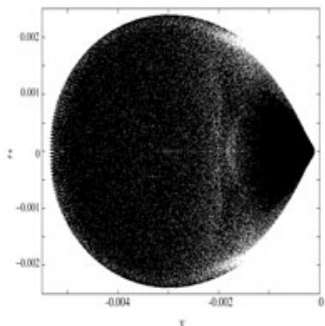

(c1)

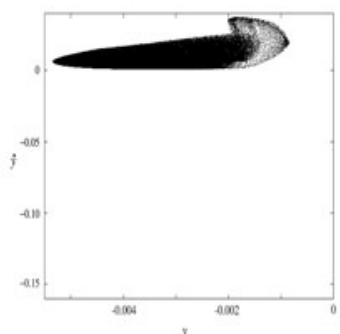

(a2)

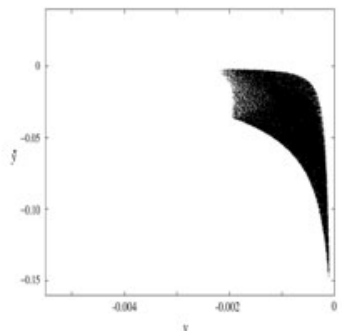

(b2)

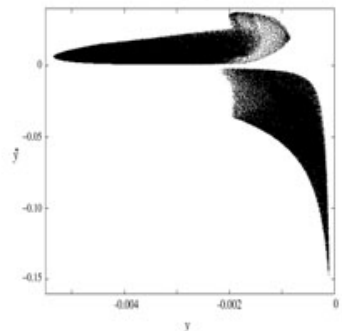

(c2)

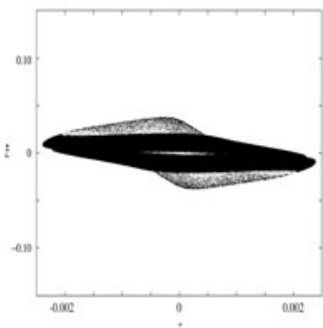

(a3)

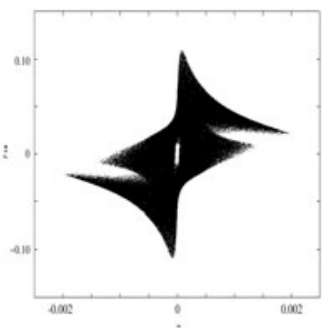

(b3)

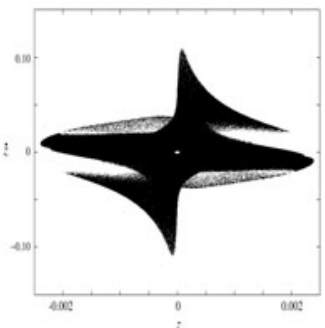

(c3)

Figure 15. Projections of $(a) \mathcal{C}_{1}^{-u 1},(b) \mathcal{C}_{1}^{-s 2}$, and (c) their superposition for Jacobi constant $C=3.00082645904328$. See the text for explanation. $(a 1)-(a 2)-(a 3):(y, z),(y, \dot{y})$, and $(z, \dot{z})$ projections associated with the $L_{1}$ point. $(b 1)-(b 2)-(b 3):(y, z),(y, \dot{y})$ and $(z, \dot{z})$ projections associated with the $L_{2}$ point. $(c 1)-(c 2)-(c 3)$ : superposition of the above figures. The set $\mathcal{I}_{1^{-}}=\mathcal{C}_{1}^{-u 1} \cap \mathcal{C}_{1}^{-s 2}$ is empty.

one point, with another orbit in the stable manifold of a libration orbit around the other point. That is, both orbits have to be the same. Since these orbits, when considered in the conventional CR3BP coordinate system, have to go from one side of the Earth to the other, the place where we look for the connection is the Poincare section defined by $x=1-\mu, \dot{x}>0$, which is orthogonal to the $x$-axis and passes through the centre of the Earth.

Although the technical details are more complex, the main idea is similar to the computations introduced in [14] for $L_{4,5}$ connections. Once a Jacobi constant is fixed, we take initial conditions in the linear approximation of the unstable manifold of all the libration orbits inside this level of energy. Since the energy is fixed, we have three free variables (usually $q_{2}, p_{2}$ and $q_{3}$ ). A scanning procedure in these variables is done. Since the selected orbits will leave the neighbourhood of the libration point, each initial condition in the variables $(q, p)$ is translated into CR3BP coordinates and then propagated forward in time until it crosses the plane $x=1-\mu$ with $\dot{x}>0$. To obtain orbits in the stable manifold around the other libration point in the same level of energy (CR3BP Jacobi constant), we do the same process except that the propagation is done backwards in time. 
(a)

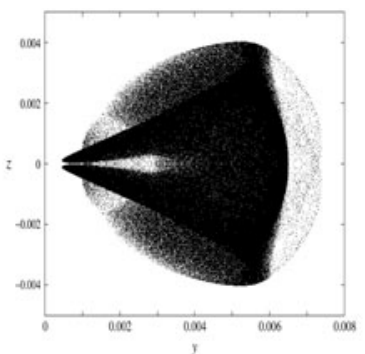

(a1)

(b)

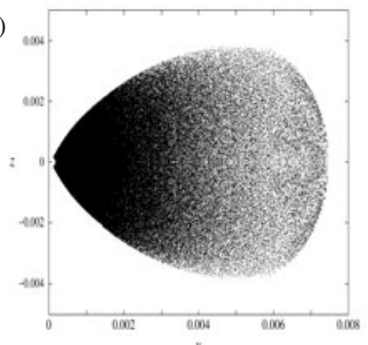

(b1)

(c)

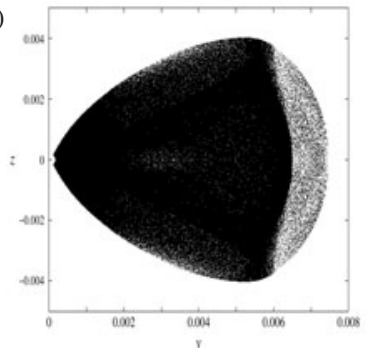

$(\mathrm{c} 1)$

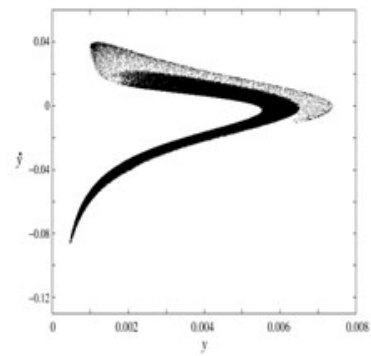

(a2)

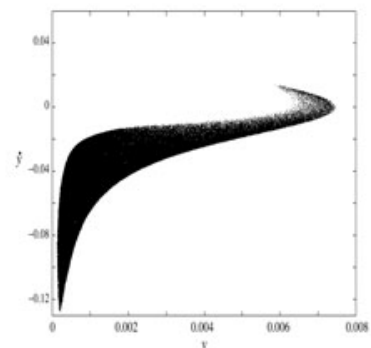

(b2)

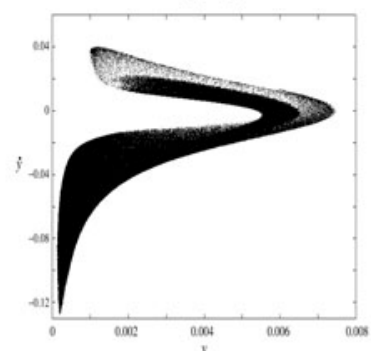

$(\mathrm{c} 2)$

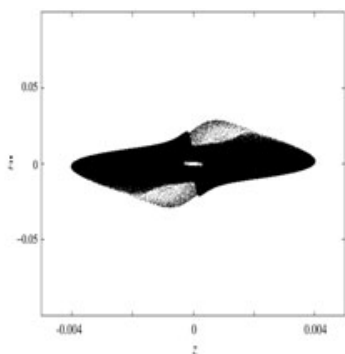

(a3)

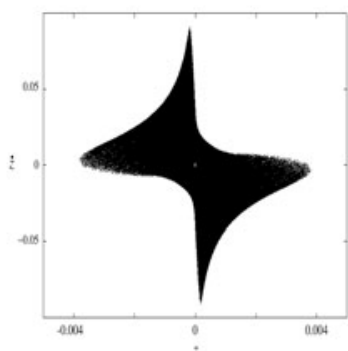

(b3)

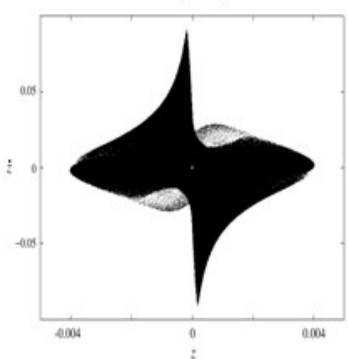

(c3)

Figure 16. Projections of $(a) \mathcal{C}_{2}^{-u 1},(b) \mathcal{C}_{2}^{-s 2}$, and (c) their superposition for Jacobi constant $C=3.00082645904328$. See the text for explanation. $(a 1)-(a 2)-(a 3):(y, z),(y, \dot{y})$, and $(z, \dot{z})$ projections associated with the $L_{1}$ point, respectively. $(b 1)-(b 2)-(b 3):(y, z),(y, \dot{y})$, and $(z, \dot{z})$ projections associated with the $L_{2}$ point, respectively. (c1)-(c2)-(c3): Superposition of the above figures. The set $\mathcal{I}_{2^{-}}=\mathcal{C}_{2}^{-u 1} \cap \mathcal{C}_{2}^{-s 2}$ is not empty.

We have to remark that, as usual, the unstable and stable manifolds have two branches. In the process we select only the Earth realm branches, i.e. those which approach $x=1-\mu$ during the initial steps of the propagation. Adopting the same labelling convention as in section 5, we compute the branches bounding left-to-right transit orbits, i.e.- -branches for $L_{1}$ and $L_{2}$. In figures 15 and 16 we show an example of the intersections of the stable manifolds of the $L_{1}$ libration orbits with the unstable manifolds of the $L_{2}$ libration orbits.

Theoretically, the simplest heteroclinic orbits will be obtained from $\mathcal{I}_{1^{-}}=\mathcal{C}_{1}^{-u 1} \cap \mathcal{C}_{1}^{-s 2}$ and $\mathcal{I}_{1^{+}}=\mathcal{C}_{1}^{+s 1} \cap \mathcal{C}_{1}^{+u 2}$. Both sets give transfer orbits that cross the plane $x=1-\mu, \dot{x}>0$ exactly once. We will denote by $\mathcal{I}_{k^{-}}$(respectively $\mathcal{I}_{k^{+}}$) the set of heteroclinic trajectories from $L_{1}$ to $L_{2}$ (respectively from $L_{2}$ to $L_{1}$ ) that cross the plane $x=\mu-1, \dot{x}>0$ exactly $k$ times, following the above-mentioned branches of the manifold. Due to the symmetries of the CR3BP equations, for any heteroclinic orbit from $L_{1}$ to $L_{2}$ we have a symmetrical one from $L_{2}$ to $L_{1}$. Therefore, we need only explore the $L_{1}$ to $L_{2}$ connecting orbits, i.e. the set $\mathcal{I}_{k^{-}}$. 

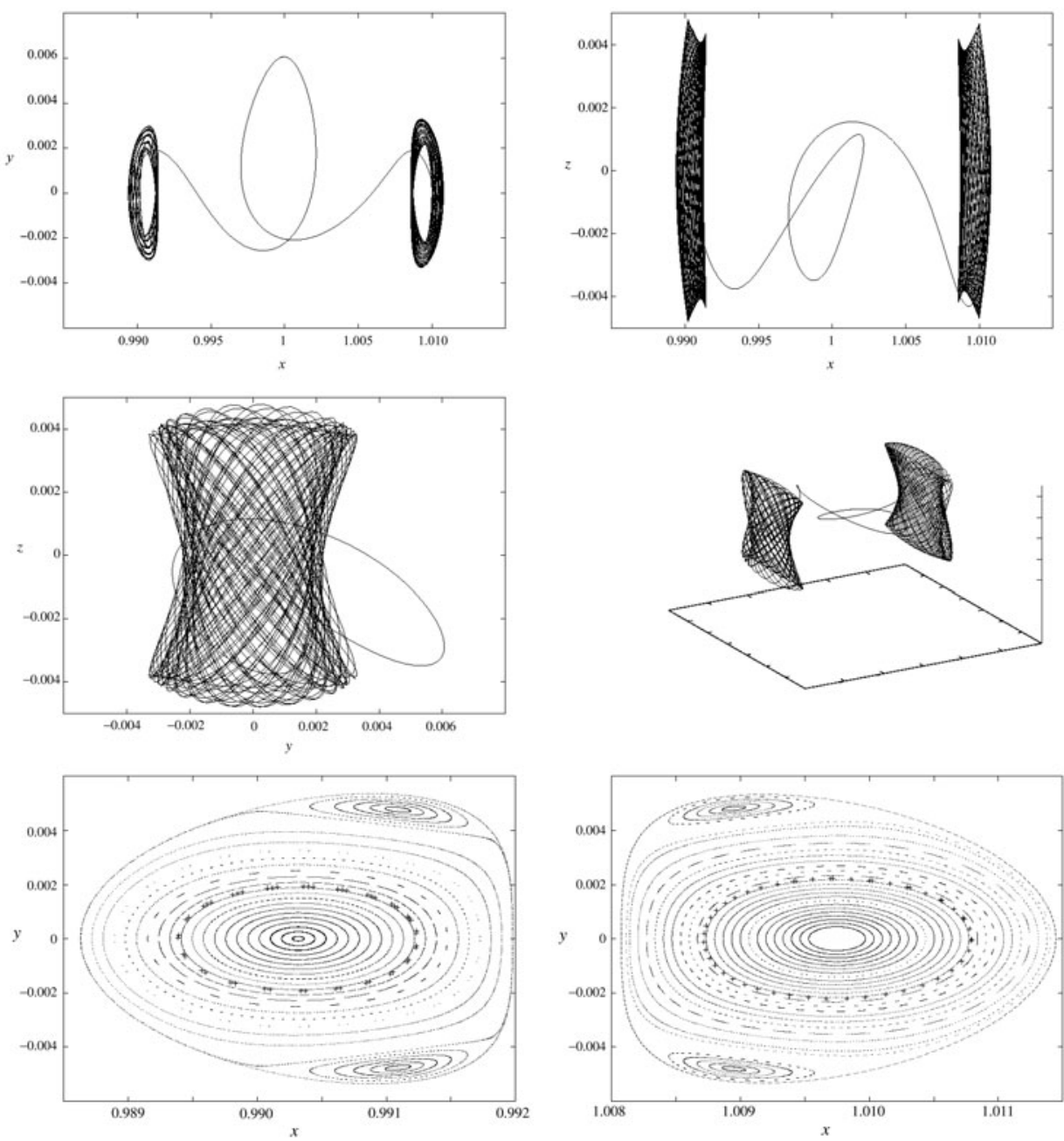

Figure 17. An $L_{1}-L_{2}$ heteroclinic connection between Lissajous orbits. In the lower pictures the intersections of the orbits with the surface of section $(z=0)$ for $L_{1}$ (left) and for $L_{2}$ (right) are displayed with crosses.

Unfortunately, as can be seen in figure 15 , the set $\mathcal{I}_{1^{-}}$is empty and so we must look for connections crossing the plane $x=1-\mu, \dot{x}>0$ twice, i.e. $\mathcal{I}_{2^{-}}$. As it turns out, $\mathcal{I}_{2^{-}} \neq \emptyset$; many possibilities of connections appear, as can be seen in figure 16. The Jacobi constant selected corresponds to a halo orbit of normalized $z$ amplitude 0.2 according to the notation used in [18] and it corresponds to the energy of the lower left pictures in figures 13 and 14 $(C=3.00080291513364)$.

Some examples of connections. In figures 17-19, two connections between Lissajous orbits around $L_{1}$ and $L_{2}$ are displayed. Both the coordinate projections and the three-dimensional representation of the heteroclinic orbits are shown. Also, the corresponding intersections with the surface of section $z=0$, around both equilibrium points, are displayed.

We have also computed a trajectory very close to a homoclinic point of the planar Lyapunov orbit inside the centre manifold. As we previously remarked, these points are between the zones 

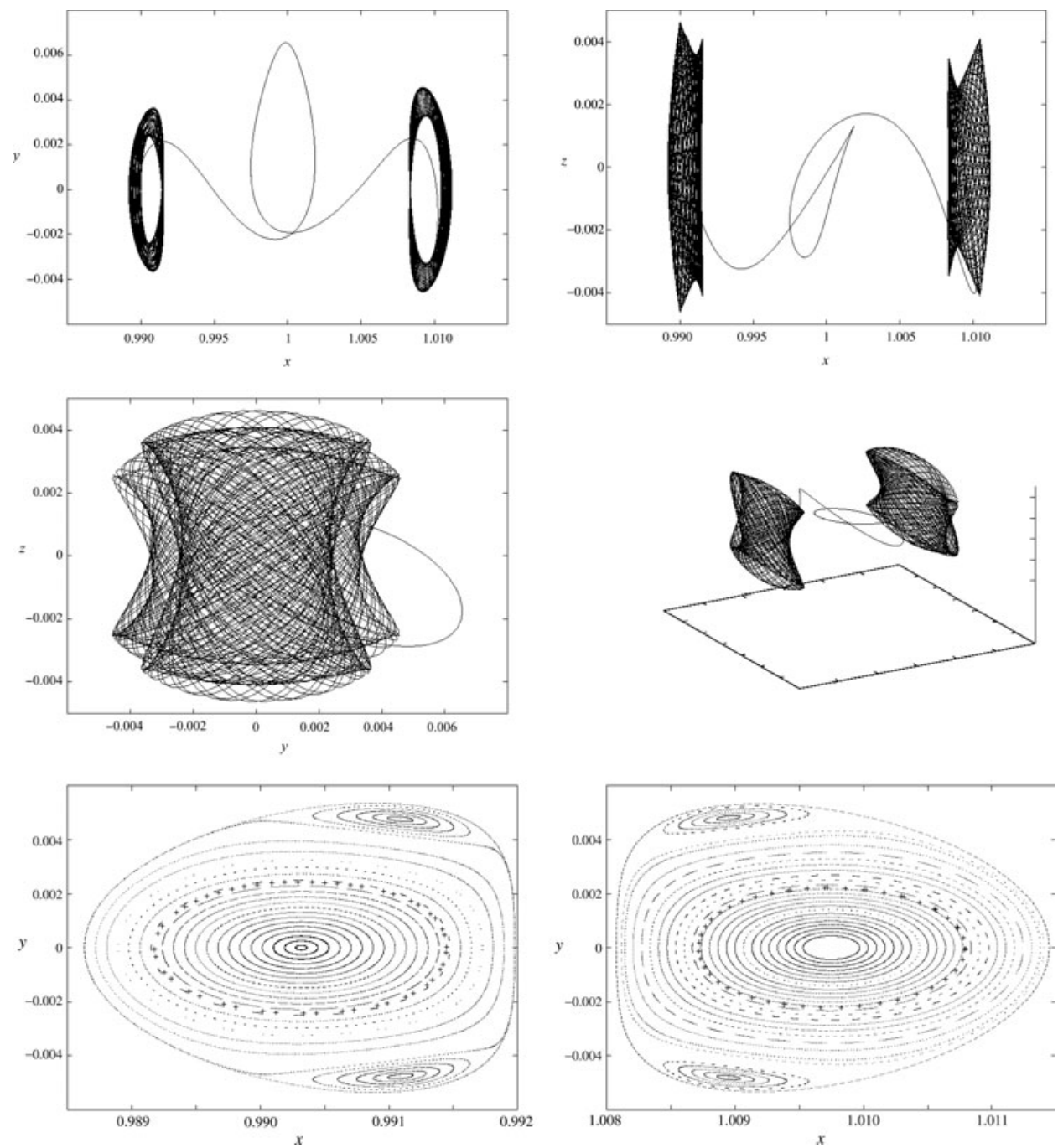

Figure 18. An $L_{1}-L_{2}$ heteroclinic connection between Lissajous orbits. In the lower pictures the intersections of the orbits with the surface of section $(z=0)$ for $L_{1}$ (left) and for $L_{2}$ (right) are displayed with crosses.

of central Lissajous and quasi-halo ones. These kinds of solutions are interesting because they perform a transition from a planar motion (close to a Lyapunov orbit) to an inclined orbit (close to the quasi-halo orbits) without any $\Delta V$. Figure 20 shows one of these orbits in central manifold $(q, p)$ variables. In figure 21 we see the variation of its $z$-amplitude. When the $z$-amplitude is close to zero the trajectory moves close to the planar Lyapunov orbit. Then, it gets close to the unstable manifold of the Lyapunov and escapes from it reaching a large $z$-amplitude during the transition. Finally, the orbit comes back close to the planar Lyapunov orbit getting close to its stable manifold. The numerical integration is performed for a long time interval and this pattern repeats several times. Unfortunately, the transition from zero $z$-amplitude to large $z$-amplitude is very slow for practical purposes. But probably, with very small $\Delta V$, it could be possible to accelerate it and have useful and cheap transitions from planar to inclined motions. 

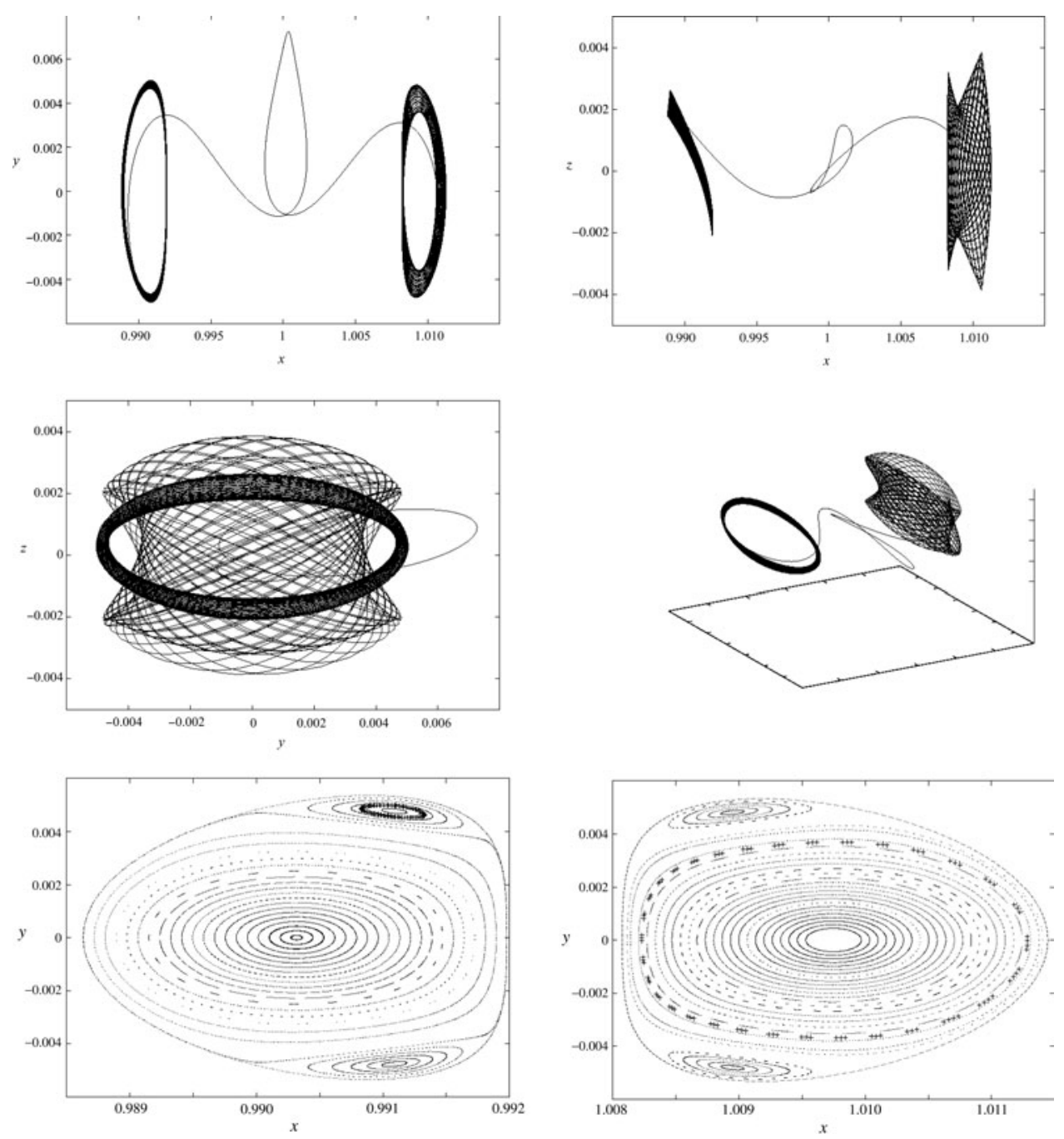

Figure 19. An $L_{1}-L_{2}$ heteroclinic connection between a Lissajous orbit around $L_{2}$ and a quasihalo orbit around $L_{1}$. In the lower pictures the intersections of the orbits with the surface of section $(z=0)$ for $L_{1}$ (left) and for $L_{2}$ (right) are shown by crosses.

\section{Conclusion and future work}

We have shown that the invariant manifold structures of the collinear libration points of the spatial three-body problem act as the separatrices between two types of motion, those inside the invariant manifold tubes are transit orbits and those outside the tubes are non-transit orbits. We have also designed a numerical algorithm for constructing orbits with any prescribed finite itinerary in the spatial three-body planet-moon-spacecraft problem. As our example, we have shown how to construct a spacecraft orbit with the basic itinerary $(X ; M, I)$.

Furthermore, we have applied the techniques developed in this paper towards the construction of a three-dimensional Petit Grand Tour of the Jovian moon system. Fortunately, the delicate dynamics of the Jupiter-Europa-Ganymede-spacecraft four-body problem are well approximated by considering it as two three-body subproblems. One can seek intersections 

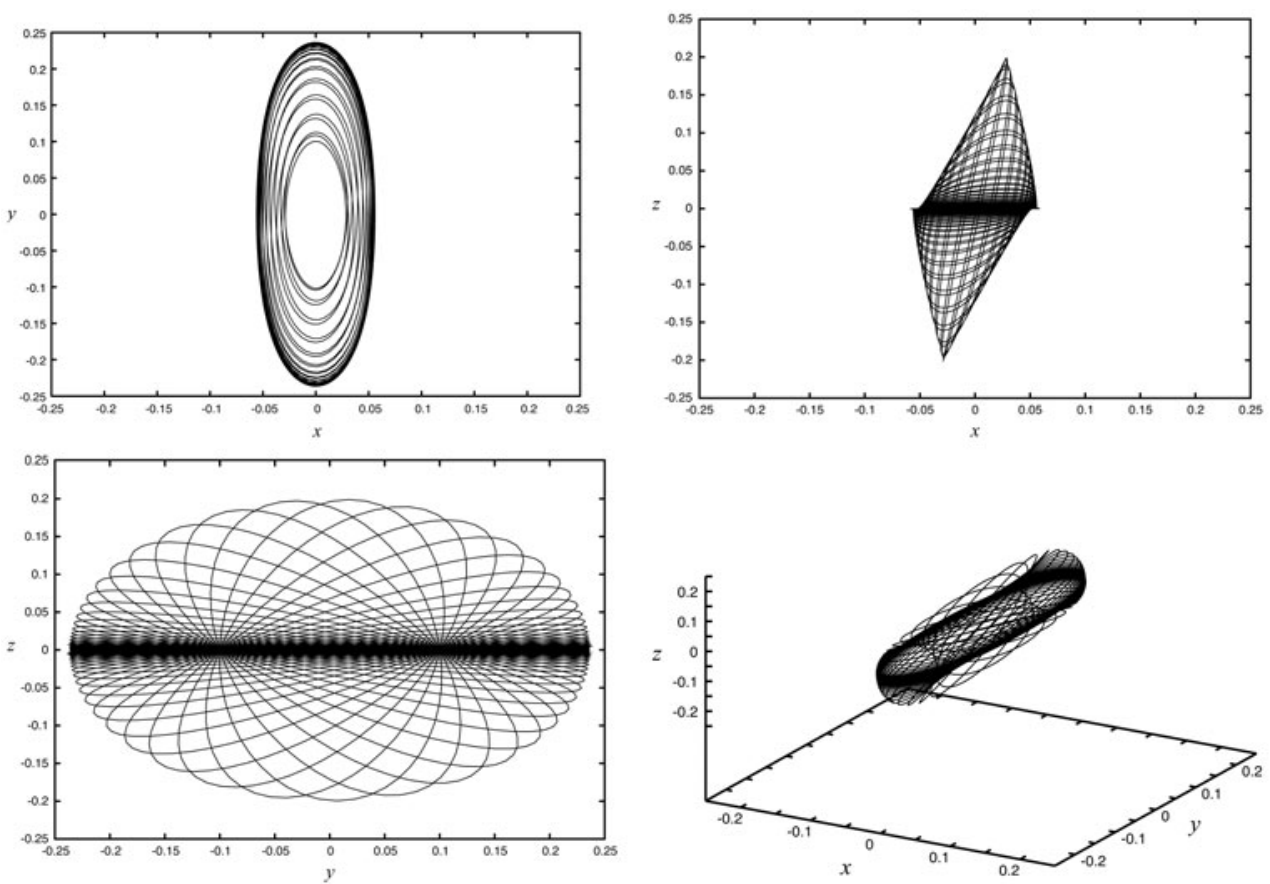

Figure 20. Homoclinic connection between Lyapunov orbits inside the central manifold (in centre manifold coordinates).

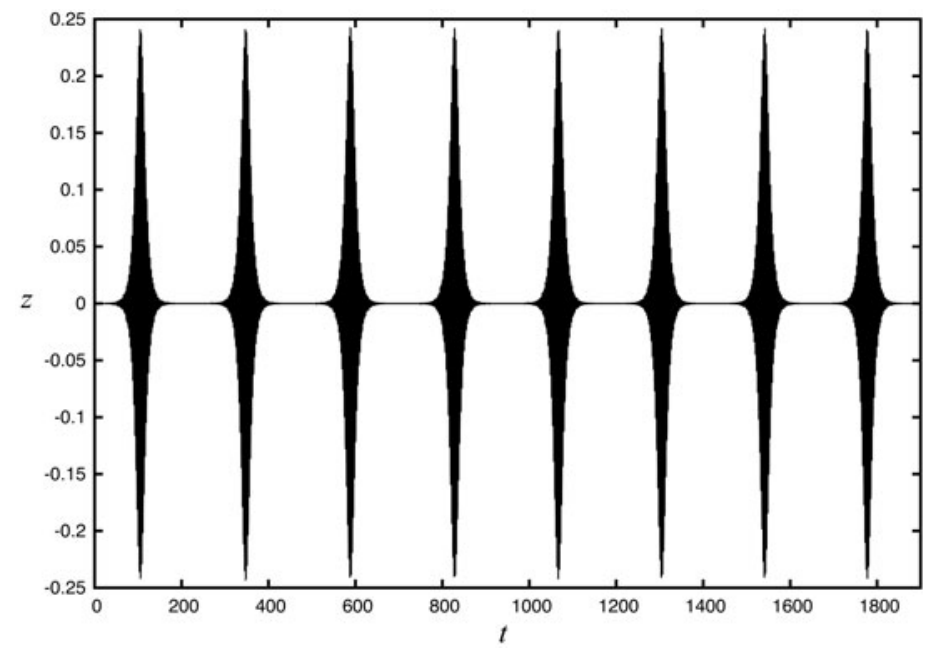

Figure 21. CR3BP time versus centre manifold $z$-amplitude for the orbit of figure 20 . See explanation in the text.

between the channels of transit orbits enclosed by the stable and unstable manifold tubes of the NHIM of different moons using the method of Poincaré sections. With manoeuvre sizes (i.e. $\Delta V$ ) much smaller than those necessary for Hohmann transfers, transfers between moons are possible. In addition, the three-dimensional details of the encounter of each moon can be controlled. In our example, we designed a trajectory that ends in a high inclination orbit 
around Europa. In the future, we would like to explore the possibility of injecting into orbits of all inclinations.

We also present a new methodology to compute the homoclinic and heteroclinic orbits, joining libration point orbits around the collinear equilibrium points $L_{1}$ and $L_{2}$ for the Sun-Earth system. The explorations reveal that the connections are a large set, and some examples are presented. For future work we expect to combine the procedure with continuation techniques in order to have a global description of the homoclinic and heteroclinic orbits for a larger range of energy values and mass ratios.

\section{Acknowledgments}

This work was carried out in part at the Jet Propulsion Laboratory and the California Institute of Technology under a contract with the National Aeronautics and Space Administration. In addition, the work was partially supported by the Caltech President's Fund, the NSF/ITR Grant ACI-0204932, the Spanish Grant BFM2000-09054 and the Catalan grant CIRIT 2001-70.

\section{Appendix: Computation of NHIM and its stable and unstable manifolds}

We have included in this appendix a brief description of the theoretical basis and the practical steps for developing the software used in the numerical explorations for this paper (for more details, see [23]).

The Hamiltonian. From the work of earlier sections, the Hamiltonian has the form

$$
H=\frac{1}{2}\left(p_{x}^{2}+p_{y}^{2}+p_{z}^{2}\right)+y p_{x}-x p_{y}-\left(\frac{1-\mu}{r_{1}}+\frac{\mu}{r_{2}}\right) .
$$

After a translation to libration point centred coordinates, with the distances scaled to the secondary and libration point distance, we wish to compute a high order expansion of the resulting Hamiltonian, which for simplicity of notation, we shall also refer to as $H$. It has the form

$$
H=\frac{1}{2}\left(p_{x}^{2}+p_{y}^{2}+p_{z}^{2}\right)+y p_{x}-x p_{y}-\sum_{n \geqslant 2} c_{n}(\mu) \rho^{n} P_{n}\left(\frac{x}{\rho}\right)
$$

where $\rho^{2}=x^{2}+y^{2}+z^{2}$ and $P_{n}$ is the Legendre polynomial of degree $n$. The coefficients $c_{n}$ are given by

$$
c_{n}=\frac{1}{\gamma_{j}^{3}}\left(( \pm 1)^{n} \mu+(-1)^{n} \frac{(1-\mu) \gamma_{j}^{n+1}}{\left(1 \mp \gamma_{j}\right)^{n+1}}\right), \quad \text { for } L_{j}, \quad j=1,2,
$$

where $\gamma_{j}$ is the distance between $L_{j}$ and the second primary. As usual, the upper sign is for $L_{1}$ and the lower one for $L_{2}$. A good way of implementing this expansion on a computer is to take advantage of the recurrence of the Legendre polynomials (see, for instance, $[10,24])$.

Linear behaviour and nonlinear expansion. The linearization of the Hamiltonian around $L_{1,2}$ given in section 3 shows that the local behaviour near these points is of the type 
saddle $\times$ centre $\times$ centre. So, using a real linear and symplectic change of coordinates, it is easy to cast the second-order part of the Hamiltonian

$$
H_{2}=\frac{1}{2}\left(p_{x}^{2}+p_{y}^{2}\right)+y p_{x}-x p_{y}-c_{2} x^{2}+\frac{c_{2}}{2} y^{2}+\frac{1}{2} p_{z}^{2}+\frac{c_{2}}{2} z^{2},
$$

into its real normal form,

$$
H_{2}=\lambda x p_{x}+\frac{\nu}{2}\left(y^{2}+p_{y}^{2}\right)+\frac{\omega}{2}\left(z^{2}+p_{z}^{2}\right) .
$$

Here, $\lambda, v$, and $\omega$ are positive real numbers given by

$$
\lambda^{2}=\frac{c_{2}-2+\sqrt{9 c_{2}^{2}-8 c_{2}}}{2}, \quad v^{2}=\frac{2-c_{2}+\sqrt{9 c_{2}^{2}-8 c_{2}}}{2}, \quad \omega^{2}=c_{2} .
$$

Note that, for simplicity, we have kept in equation (A1) the same notation for the variables even after a coordinate change.

For the following normal form computations it is convenient to 'diagonalize' the second-order terms. This is done by introducing the complex change of coordinates:

$$
\begin{aligned}
& \left(\begin{array}{c}
y \\
p_{y}
\end{array}\right)=\frac{1}{\sqrt{2}}\left(\begin{array}{cc}
1 & \sqrt{-1} \\
\sqrt{-1} & 1
\end{array}\right)\left(\begin{array}{l}
q_{2} \\
p_{2}
\end{array}\right), \\
& \left(\begin{array}{c}
z \\
p_{z}
\end{array}\right)=\frac{1}{\sqrt{2}}\left(\begin{array}{cc}
1 & \sqrt{-1} \\
\sqrt{-1} & 1
\end{array}\right)\left(\begin{array}{l}
q_{3} \\
p_{3}
\end{array}\right)
\end{aligned}
$$

and re-naming $x=q_{1}$ and $p_{x}=p_{1}$, the second-order part of the Hamiltonian becomes

$$
H_{2}=\lambda q_{1} p_{1}+\sqrt{-1} v q_{2} p_{2}+\sqrt{-1} \omega q_{3} p_{3}
$$

From now on we will use the following notation. If $x=\left(x_{1}, \ldots, x_{n}\right)$ is a vector of complex numbers and $k=\left(k_{1}, \ldots, k_{n}\right)$ is an integer vector, we denote by $x^{k}$ the term $x_{1}^{k_{1}} \cdots x_{n}^{k_{n}}$ (in this context we define $0^{0}$ as 1$)$. Moreover, we define $|k|$ as $\sum_{j}\left|k_{j}\right|$.

In order to have all possible orbits in the centre manifold, let us expand the initial Hamiltonian $H$ using the coordinates that give us $H_{2}$ as in (A3). Then, the expanded Hamiltonian takes the form

$$
H(q, p)=H_{2}(q, p)+\sum_{n \geqslant 3} H_{n}(q, p)
$$

where $H_{2}$ is given in (A3) and $H_{n}$ denotes a homogeneous polynomial of degree $n$ of the form $\sum_{i, j} h_{i j} q_{1}^{i_{1}} p_{1}^{j_{1}} q_{2}^{i_{2}} p_{2}^{j_{2}} q_{3}^{i_{3}} p_{3}^{j_{3}}$, where $h_{i j}$ denotes $h_{i_{1}, i_{2}, i_{3}, j_{1}, j_{2}, j_{3}}$.

Review of normal form computation. The process of reduction to the centre manifold is similar to a normal form computation. The objective is to remove some monomials in the expansion of the Hamiltonian, in order to have an invariant manifold tangent to the centre directions of $\mathrm{H}_{2}$. For this purpose, let us recall that, if $F(q, p)$ and $G(q, p)$ are two functions of positions, $q$, and momenta, $p$, their Poisson bracket is defined as

$$
\{F, G\}=\sum_{i=1}^{3}\left(\frac{\partial F}{\partial q_{i}} \frac{\partial G}{\partial p_{i}}-\frac{\partial F}{\partial p_{i}} \frac{\partial G}{\partial q_{i}}\right) .
$$

The changes of variables are implemented by means of the Lie series method with some similarity to [6]. If $G(q, p)$ is a Hamiltonian system, then the function $\hat{H}$ defined by

$$
\hat{H} \equiv H+\{H, G\}+\frac{1}{2 !}\{\{H, G\}, G\}+\frac{1}{3 !}\{\{\{H, G\}, G\}, G\}+\cdots,
$$


is the result of applying a canonical change to $H$. This change is the time one flow corresponding to the Hamiltonian $G . G$ is usually called the generating function of the transformation (A5). See [8] and references therein for more theoretical details. Here, we will explain only the basics of the procedure.

Note that if $P$ and $Q$ are two homogeneous polynomials of degree $r$ and $s$, respectively, then $\{P, Q\}$ is a homogeneous polynomial of degree $r+s-2$. This means that if $G_{3}$ is a homogeneous polynomial of degree 3 used as a generating function, then the homogeneous polynomials of degree $n, \hat{H}_{n}$, such that $\hat{H}=\sum_{n \geqslant 2} \hat{H}_{n}$, are given by,

$$
\begin{aligned}
& \hat{H}_{2}=H_{2}, \\
& \hat{H}_{3}=H_{3}+\left\{H_{2}, G_{3}\right\}, \\
& \hat{H}_{4}=H_{4}+\left\{H_{3}, G_{3}\right\}+\frac{1}{2 !}\left\{\left\{H_{2}, G_{3}\right\}, G_{3}\right\} . \\
& \ldots \ldots \ldots
\end{aligned}
$$

If we are interested in removing all the terms of order three in the new Hamiltonian, i.e. to have $\hat{H}_{3}=0$, we must choose $G_{3}$ such that it solves the homological equation $\left\{H_{2}, G_{3}\right\}=-H_{3}$.

This procedure can be used recurrently trying to find a homogeneous polynomial of degree four, $G_{4}$, to remove all the terms of order four of the new Hamiltonian, $\hat{H}$, and so on. Nevertheless, we must point out that this is not always possible and some resonant terms, even of order four, cannot be cancelled. Anyway, this process is used to compute what is known as the Birkhoff normal form of the Hamiltonian, having the minimum number of monomials up to some degree.

We note that a Lie transformation method is used for a similar Hamiltonian system describing an atomic system in [40] to obtain a normal form up to order 6. However, there are notable differences in the implementation: they use the Lie triangle, which we do not. Moreover, the type of normal form we seek is different. The reduction to the centre manifold which we use is just a certain type of normal form, characterized for 'killing' certain monomials, which we find convenient for our purposes.

Reduction to the centre manifold. Although the reduction to the centre manifold is based on this scheme, we only need to remove the instability associated with the hyperbolic character of the Hamiltonian $H$. We note that the second-order part of the Hamiltonian $\mathrm{H}_{2}$ gives us the linear part of the Hamiltonian equations, and so, the instability is associated with the term $\lambda q_{1} p_{1}$. For this linear approximation of the Hamiltonian equations, the centre part can be obtained by setting $q_{1}=p_{1}=0$. If we want the trajectory to remain tangent to this space (i.e. to have $q_{1}(t)=p_{1}(t)=0$ for all $t>0$ ), then we need to have $\dot{q}_{1}(0)=\dot{p}_{1}(0)=0$ when adding the nonlinear terms. Due to the autonomous character of the Hamiltonian system, we will obtain $q_{1}(t)=p_{1}(t)=0$ for all $t \geqslant 0$.

Recalling that the Hamiltonian equations associated with a Hamiltonian $H(q, p)$ are

$$
\dot{q}_{i}=\frac{\partial H}{\partial p_{i}}, \quad \dot{p}_{i}=-\frac{\partial H}{\partial q_{i}},
$$

in particular,

$$
\begin{aligned}
& \dot{q}_{1}=\frac{\partial H}{\partial p_{1}}=\lambda q_{1}+\sum_{n \geqslant 3} h_{i j} q_{1}^{i_{1}} p_{1}^{j_{1}-1} q_{2}^{i_{2}} p_{2}^{j_{2}} q_{3}^{i_{3}} p_{3}^{j_{3}}, \\
& \dot{p}_{1}=-\frac{\partial H}{\partial q_{1}}=-\lambda p_{1}-\sum_{n \geqslant 3} h_{i j} q_{1}^{i_{1}-1} p_{1}^{j_{1}} q_{2}^{i_{2}} p_{2}^{j_{2}} q_{3}^{i_{3}} p_{3}^{j_{3}},
\end{aligned}
$$


one can get the required condition, $\dot{q}_{1}(0)=\dot{p}_{1}(0)=0$ when $q_{1}(0)=p_{1}(0)=0$, if in the series expansion of the Hamiltonian $H$, all the monomials, $h_{i j} q^{i} p^{j}$, with $i_{1}+j_{1}=1$, have $h_{i j}=0$. This happens if there are no monomials with $i_{1}+j_{1}=1$. Since this minimalist expansion needs to cancel fewer monomials in (A4), in principle, it may be better behaved both in terms of convergence and from a numerical point of view. Of course other expansions could give us the same required tangency, such as the one which kills all the monomials with $i_{1} \neq j_{1}$. This alternative expansion gives an approximated first integral and can be useful for theoretical purposes.

All the computations discussed above have been implemented by writing specific symbolic manipulators in Fortran that can carry out all the procedures up to an arbitrary order. For practical purposes, and in order to have an acceptable equilibrium between precision and time computing requirements, the normal form scheme has been implemented up to order $N=15$.

After all these changes of variables, the initial complexified Hamiltonian around the collinear libration points has been expanded in the following form

$$
H(q, p)=\bar{H}_{N}(q, p)+R_{N}(q, p),
$$

where $\bar{H}_{N}(q, p)$ is a polynomial of degree $N$ without terms of $i_{1}+j_{1}=1$ in the minimalist case, or without terms of $i_{1} \neq j_{1}$ in the first integral case. $R_{N}(q, p)$ is a remainder of order $N+1$, which is very small near $L_{1,2}$ and will be skipped in further computations.

Finally, using the inverse change of variables of (A2), the truncated Hamiltonian $\bar{H}_{N}$ can be expanded in real form and we obtain

$$
\bar{H}_{N}(q, p)=H_{2}(q, p)+\sum_{n=3}^{N} H_{n}(q, p),
$$

where, explicitly,

$$
H_{2}(q, p)=H_{2}\left(q_{1}, p_{1}, q_{2}, p_{2}, q_{3}, p_{3}\right)=\lambda q_{1} p_{2}+\frac{v}{2}\left(q_{2}^{2}+p_{2}^{2}\right)+\frac{\omega}{2}\left(q_{3}^{2}+p_{3}^{2}\right) .
$$

For convenience, the variables are called $q, p$ again.

NHIM and its stable and unstable manifolds. As discussed previously, in the case when all the monomials in $H_{n}$ with $i_{1} \neq j_{1}$ have been eliminated, the truncated Hamiltonian $\bar{H}_{N}$ has a first integral, $I=q_{1} p_{1}$. This is because $\bar{H}_{N}$ is given by

$$
\bar{H}_{N}=H_{2}\left(I, q_{2}, p_{2}, q_{3}, p_{3}\right)+\sum_{n=3}^{N} H_{n}\left(I, q_{2}, p_{2}, q_{3}, p_{3}\right) .
$$

Let $f$ be a function of the centre manifold variables $\left(q_{2}, p_{2}, q_{3}, p_{3}\right)$ defined as

$$
f\left(q_{2}, p_{2}, q_{3}, p_{3}\right)=\sum_{n=3}^{N} H_{n}\left(0, q_{2}, p_{2}, q_{3}, p_{3}\right) .
$$

Then, $f$ is at least of third order. Note that the invariant manifold $\mathcal{S}_{h}$ defined by

$\mathcal{S}_{h}=\left\{(q, p) \mid \frac{v}{2}\left(q_{2}^{2}+p_{2}^{2}\right)+\frac{\omega}{2}\left(q_{3}^{2}+p_{3}^{2}\right)+f\left(q_{2}, p_{2}, q_{3}, p_{3}\right)=h, q_{1}=p_{1}=0\right\}$

is the NHIM for the nonlinear system which corresponds to the 3-sphere (6) for the linearized system. In a small neighbourhood of the equilibrium point, since the higher order terms in $f$ are much smaller than the second-order terms, the 3-sphere for the linear problem becomes a 
deformed sphere for the nonlinear problem. Moreover, since NHIMs persist under perturbation, this deformed sphere $\mathcal{S}_{h}$ still has stable and unstable manifolds which are given by

$$
\begin{aligned}
& W_{ \pm}^{s}\left(\mathcal{S}_{h}\right)=\left\{(q, p) \mid \frac{v}{2}\left(q_{2}^{2}+p_{2}^{2}\right)+\frac{\omega}{2}\left(q_{3}^{2}+p_{3}^{2}\right)+f\left(q_{2}, p_{2}, q_{3}, p_{3}\right)=h, q_{1}=0\right\}, \\
& W_{ \pm}^{u}\left(\mathcal{S}_{h}\right)=\left\{(q, p) \mid \frac{v}{2}\left(q_{2}^{2}+p_{2}^{2}\right)+\frac{\omega}{2}\left(q_{3}^{2}+p_{3}^{2}\right)+f\left(q_{2}, p_{2}, q_{3}, p_{3}\right)=h, p_{1}=0\right\} .
\end{aligned}
$$

Note the similarity between the formulae above and those for the linearized problem given in equations (7) and (8), especially given the fact that these two coordinate systems are linked by a near-identity transformation.

\section{References}

[1] Appleyard D F 1970 Invariant sets near the collinear Lagrangian points of the nonlinear restricted three-body problem $P h D$ Thesis University of Wisconsin

[2] Belbruno E A and Miller J K 1993 Sun-perturbed Earth-to-Moon transfer with ballistic capture J. Guidance Control Dyn. $16770-5$

[3] Conley C 1968 Low energy transit orbits in the restricted three-body problem SIAM J. Appl. Math. 16 732-46

[4] Delshams A and Gutiérrez P 1999 Homoclinic orbits to invariant tori in Hamiltonian systems Multilple-TimeScale Dynamical Systems (IMA Volumes in Mathematics and its Applications) ed C Jones et al (New York: Springer)

[5] Delshams A, Gutiérrez P and Seara T M 2004 Exponentially small splitting for whiskered tori in Hamiltonian systems: flow-box coordinates and upper bounds Discrete Continuum Dyn. Syst. at press

[6] Deprit A 1969 Canonical transformations depending on a small parameter Celestial Mech. 1 12-30

[7] Farquhar R W, Muhonen D P, Newman C and Heuberger H 1979 The first libration point satellite, mission overview and flight history AAS/AIAA Astrodynamics Specialist Conf. (Provincetown, MA)

[8] Giorgilli A, Delshams A, Fontich E, Galgani L and Simó C 1989 Effective stability for a Hamiltonian system near an elliptic equilibrium point with an application to the restricted three body problem J. Diff. Eqns 77 167-98

[9] Gómez G, Howell K C, Masdemont J and Simó C 1998 Station-keeping strategies for translunar libration point orbits Adv. Astronaut. Sci. 99 949-67

[10] Gómez G, Jorba À, Masdemont J and Simó C 1991 Study refinement of semianalytical halo orbit theory Final Report ESOC Contract No 8625/89/D/MD(SC)

[11] Gómez, G, Jorba À, Masdemont J and Simó C 1998 Study of the transfer between halo orbits Acta Astronaut. $43493-520$

[12] Gómez G, Jorba À, Masdemont J and Simó C 2001 Dynamics and mission design near libration points Advanced Methods for Collinear Points vol III (Singapore: World Scientific)

[13] Gómez G, Koon W S, Lo M W, Marsden J E, Masdemont J and Ross S D 2001 Invariant manifolds, the spatial three-body problem and space mission design Adv. Astronaut. Sci. 109 3-22 AAS paper 01-301

[14] Gómez G, Llibre J and Masdemont J 1988 Homoclinic and heteroclinic solutions in the restricted three-body problem Celestial Mech. 44 239-59

[15] Gómez G and Masdemont J 2000 Some zero cost transfers between libration point orbits AAS/AIAA Astrodynamics Specialist Conf. (Florida) paper AAS 00-177

[16] Gómez G, Masdemont J and Simó C 1993 Study of the transfer from the Earth to a halo orbit around the equilibrium point $L_{1}$ Celestial Mech. Dyn. Astron. 56 541-62

[17] Gómez G, Masdemont J and Simó C 1997 Lissajous orbits around halo orbits Adv. Astronaut. Sci. 95 117-34

[18] Gómez G, Masdemont J and Simó C 1998 Quasihalo orbits associated with libration points J. Astronaut. Sci. 46 135-76

[19] Hartman P 1964 Ordinary Differential Equations (New York: Wiley)

[20] Howell K, Barden B and Lo M 1997 Application of dynamical systems theory to trajectory design for a libration point mission J. Astronaut. Sci. 45 161-78

[21] Howell K C, Barden B T, Wilson R S and Lo M W 1997 Trajectory design using a dynamical systems approach with application to genesis AAS/AIAA Astrodynamics Specialist Conf. (Sun Valley, Idaho) AAS paper 97-709

[22] Jaffé C, Ross S D, Lo M W, Marsden J E, Farrelly D and Uzer T 2002 Statistical theory of asteroid escape rates Phys. Rev. Lett. 89011101

[23] Jorba A and Masdemont J 1999 Dynamics in the center manifold of the collinear points of the restricted three body problem Physica D 132 189-213 
[24] Jorba À and Simó C 1994 Effective stability for periodically perturbed Hamiltonian systems Hamiltonian Mechanics, Integrability and Chaotic Behavior ed J Seimenis (New York: Plenum) pp 245-52

[25] Jorba À and Villanueva J 1997 On the normal behaviour of partially elliptic lower dimensional tori of Hamiltonian systems Nonlinearity $10427-73$

[26] Koon W S, Lo M W, Marsden J E and Ross S D 1999 The Genesis trajectory and heteroclinic connections AAS/AIAA Astrodynamics Specialist Conf. (Girdwood, Alaska) AAS paper 99-451

[27] Koon W S, Lo M W, Marsden J E and Ross S D 2000 Heteroclinic connections between periodic orbits and resonance transitions in celestial mechanics Chaos 10 427-69

[28] Koon W S, Lo M W, Marsden J E and Ross S D 2001 Resonance and capture of Jupiter comets Celestial Mech. Dyn. Astron. 81 27-38

[29] Koon W S, Lo M W, Marsden J E and Ross S D 2001 Low energy transfer to the Moon Celestial Mech. Dyn. Astron. 81 63-73

[30] Koon W S, Lo M W, Marsden J E and Ross S D 2002 Constructing a low energy transfer between Jovian moons Contemp. Math., Am. Math. Soc. 292 129-45

[31] Ludwinski J, Guman M, Johannesen J, Mitchell R and Staehle R 1998 The Europa orbiter mission design 49th Int. Astronautical Congr. (Melbourne, Australia, 28 September-2 October 1998) paper no 98-4.2.02

[32] Marsden J E and Ratiu T S 1999 Introduction to Mechanics and Symmetry (Texts in Applied Mathematics vol 17, 2nd edn) (Berlin: Springer)

[33] McGehee R P 1969 Some homoclinic orbits for the restricted three-body problem PhD Thesis University of Wisconsin

[34] Moser J 1958 On the generalization of a theorem of A Lyapunov Commun. Pure Appl. Math. XI 257-71

[35] Richardson D L 1980 A note on a Lagrangian formulation for motion about the collinear points Celestial Mech. 22 231-6

[36] Richardson D L 1980 Halo orbit formulation for the ISEE-3 mission J. Guidance Control 3 543-8

[37] Scheeres D J, Guman M D and Villac B 2001 Stability analysis of planetary satellite orbiters: application to the Europa orbiter J. Guidance Control Dyn. 24 778-87

[38] Sweetser T, Maddock R, Johannesen J, Bell J, Penzo P, Wolf A, Williams S, Matousek S and Weinstein S 1997 Trajectory design for a Europa orbiter mission: a plethora of astrodynamic challenges AAS/AIAA Space Flight Mechanics Meeting (Huntsville, AL, February 1997) paper no AAS 97-174

[39] Szebehely V 1967 Theory of Orbits (New York: Academic)

[40] Uzer T, Jaffé C, Palacián J, Yanguas P and Wiggins S 2002 The geometry of reaction dynamics Nonlinearity 15 957-92

[41] Villac B F and Scheeres D J 2003 Escaping trajectories in the Hill three-body problem and applications J. Guidance Control Dyn. 26 224-32

[42] Wiggins S 1994 Normally Hyperbolic Invariant Manifolds in Dynamical Systems (New York: Springer)

[43] Wiggins S, Wiesenfeld L, Jaffé C and Uzer T 2001 Impenetrable barriers in phase space Phys. Rev. Lett. 86 $5478-81$ 